\title{
ON THE FOURIER TRANSFORM OF A COMPACT SEMISIMPLE LIE GROUP
}

\author{
N. J. WILDBERGER
}

(27 May 1991)

Communicated by I. Raeburn

\begin{abstract}
We develop a concrete Fourier transform on a compact Lie group by means of a symbol calculus, or *-product, on each integral co-adjoint orbit. These $*$-products are constructed by means of a moment map defined for each irreducible representation. We derive integral formulae for these algebra structures and discuss the relationship between two naturally occurring inner products on them. A global Kirillov-type character is obtained for each irreducible representation. The case of $S U(2)$ is treated in some detail, where some interesting connections with classical spherical trigonometry are obtained.
\end{abstract}

1991 Mathematics subject classification (Amer. Math. Soc.): 22 E 46, 58 F 06.

\section{Introduction}

Classical harmonic analysis on the real line $\mathbb{R}$ and the torus $T$ is largely the study of the Fourier transform. This is a map from functions on the group $G$ to functions on the dual $\hat{G}$, the set of unitary characters of $G$. It is given by the formula

$$
\phi^{\wedge}(\chi)=\int_{G} \phi(g) \chi(g) d g
$$

where $\phi$ is a function on $G$ and $\chi \in \hat{G}$. Of course this is a formal definition whose meaning must be investigated for $\phi$ belonging to various spaces of functions (or

(C) 1994 Australian Mathematical Society 0263-6115/94 \$A2.00+0.00 
distributions) on $G$. The point here is that for these groups, the dual $\hat{G}$ is itself a topological group and the Fourier transform defined by $(*)$ is a very explicit and computable object. The resulting theory has a well-known generalisation to locally compact abelian groups. For non-abelian groups, the situation is considerably more complicated. We will restrict our attention here to real Lie groups. Then the proper definition of the dual $\hat{G}$ is the set of equivalence classes of irreducible unitary representations. One then defines the Fourier transform for $G$ by generalising (*). That is for $\phi$ a function on $G$ and $\rho \in \hat{G}$ one defines

$$
\phi^{\wedge}(\rho)=\int_{G} \phi(g) \rho(g) d g .
$$

This is then an operator-valued function of $\hat{G}$, with the operators $\phi^{\wedge}(\rho)$ acting on (generally) different Hilbert spaces for different $\rho \in \hat{G}$. As such it also really depends on our choice of representation in each equivalence class of $\hat{G}$. It is also clear that a necessary preliminary to any systematic study of the Fourier transform is a description of the dual $\hat{G}$. This turns out to be a deep and difficult problem. For example, the dual of $G=G L(n, \mathbb{R})$ has only recently been classified (see Vogan [21]), and the situation for general reductive groups is not yet understood, although progress has been made (see for example, Knapp and Speh [10]).

Even with the dual $\hat{G}$ in hand however, the abstract nature of $(* *)$ is an obstacle to further study. It may not be possible to even formulate analogues of classical results from the abelian theory in any natural way.

The purpose of this paper is to describe an alternate framework for the definition and study of the Fourier transform for $G$ a compact connected semisimple Lie group. In effect we construct a new, geometric Fourier transform $F$ for such a group and then show that it incorporates the abstract definition of (**). We may motivate this construction by going back to the Fourier transform for $\mathbb{R}$ or $T$ and rewriting (*) as

$(* * *)$

$$
\phi^{\wedge}(\chi)=\int_{G} \phi(g) e(g, \chi) d g .
$$

Here $e(g, \chi)=\chi(g)$ can be considered a function on $G \times \hat{G}$. Now for these groups $\hat{G}$ can be naturally identified with a subset of the dual of the Lie algebra $\mathfrak{g}$. That is $\hat{G} \simeq \mathfrak{g}_{\mathrm{INT}}^{*}$ where $\mathfrak{g}_{\mathrm{INT}}^{*}$ is the set of points in $\mathfrak{g}^{*}$ satisfying a certain integrality condition. Thus $e$ may be considered to be a function on $G \times \mathfrak{g}_{\mathrm{INT}}^{*}$, which is naturally a subset of the cotangent bundle $T^{*} G$ of $G$, which we may call $T^{*} G_{\mathrm{INT}}$, the integral cotangent bundle. 
The usefulness of this point of view is that even for non-abelian groups, there is a close connection between $\hat{G}$ and the dual of the Lie algebra $\mathbf{g}^{*}$. Now the group $G$ acts nontrivially on $\mathfrak{g}^{*}$ however, so it is the orbits of this action, the so-called co-adjoint orbits, that play the role of the points of $\mathfrak{g}^{*}$ in the abelian case. It is Kirillov's fundamental observation that for nilpotent connected simply-connected Lie groups, there is a natural bijection between $\hat{G}$ and the set of co-adjoint orbits (see Kirillov [9]). The theory of geometric quantization, introduced by Kostant [11] and Souriau [16], has shown that this close connection extends to many other groups. It becomes necessary, however, to introduce an integrality condition on co-adjoint orbits and thus to consider only integral co-adjoint orbits. One consequence of this theory is that for $G$ a compact connected semisimple Lie group, $\hat{G}$ is naturally in bijection with the set of integral orbits in $\mathfrak{g}^{*}$, which we call $\mathfrak{g}_{\mathrm{INT}}^{*}$. (This fact can be considered to be a restatement of the classical description of $\hat{G}$ by highest weights together with the Borel-Weil theorem.)

Now we may define as before, the integral contangent bundle $T^{*} G_{\mathrm{INT}}$ as $G \times \mathfrak{g}_{\mathrm{INT}}^{*}$. Our main result is the following. There exists on $T^{*} G_{\mathrm{INT}}$ a function $e$ which we call the Fourier kernel of $G$, which defines a Fourier transform $F$ from functions on $G$ to functions on $\mathfrak{g}_{\text {INT }}^{*}$ by

$$
F \phi(f)=\int_{G} \phi(g) e(g, f) d g
$$

for $\phi$ a function on $G, f \in \mathfrak{g}_{\mathrm{INT}}^{*}$. This Fourier transform incorporates the abstract one by means of a symbol calculus on each orbit (to be explained). The Fourier kernel is constructed in a theoretically explicit fashion, it turns out to be both unique and canonical, and in some sense contains all the representation theoretic information for the group.

To justify our terminology of Fourier transform for the map $F$, we show that it behaves in ways that resemble the abelian case. To describe this analogy in more detail requires some additional results and notation.

First of all, if $\rho \in \hat{G}$ and $\mathscr{O}_{\rho} \subset \mathfrak{g}_{\mathrm{INT}}^{*}$ is the associated integral orbit, we show that there is a canonical finite dimensional space of functions $A_{\rho}$ on $\mathscr{O}_{\rho}$, and an isomorphism

$$
a: \text { End } V_{\rho} \rightarrow A_{\rho}
$$

where $V_{\rho}$ is the space on which $\rho$ acts. The isomorphism ' $a$ ' establishes a symbol calculus for operators on End $V_{\rho}$. It also allows the algebra structure 
of End $V_{\rho}$ to be transferred to $A_{\rho}$; we call this the $*$-product on $A_{\rho}$ (or on $\mathscr{O}_{\rho}$ ). This construction generalises the $*$-products constructed on the 2 -sphere $S^{2}$ by Moreno and Ortega-Navarro [14].

Now it turns out that if $U_{\rho} \subset C(G)$ is the space of all matrix coefficients of $\rho$, then $F: U_{\rho} \rightarrow A_{\rho}$ is an isomorphism. That is, for $u \in U_{\rho}, F u$ is supported only on the orbit $\mathscr{O}_{\rho}$, where it is an element of $A_{\rho}$. Thus $F$ separates a function on $G$ into its Fourier components. Furthermore convolution in $U_{\rho}$ is taken to the $*$-product on $A_{\rho}$, so we may say that convolution on the group is taken to an orbitwise product on $\mathfrak{g}_{\mathrm{INT}}^{*}$ by $F$. We also show that the Fourier transform of a positive-definite function $u \in U_{\rho}$ is a positive function in $A_{\rho}$.

One of the most important results in the classical representation theory for $G$ is the Weyl character formula. Recently it has become well-known that this formula is equivalent to Kirillov's character formula, which states that the character $\chi_{\rho}$ of a representation $\rho \in \hat{G}$ can be obtained by 'pushing down' from $\mathfrak{g}$ the Euclidean inverse Fourier transform of the invariant measure $d \mu$ on $\mathscr{O}_{\rho}$. This 'push down' involves the exponential map as well as a factor related to its Jacobian. (For a precise description, see Khalgui [8]).

Kirillov's character formula seems to break down where the exponential map is not well- behaved. We show that the Fourier transform $F$ may be used to introduce a similar character formula which however is globally defined. The result is that

$$
F \bar{\chi}_{\rho}= \begin{cases}\left(\operatorname{dim} V_{\rho}\right)^{-1} & \text { on } \mathscr{O}_{\rho} ; \\ 0 & \text { elsewhere. }\end{cases}
$$

In other words $\bar{\chi}_{\rho}$ is the 'inverse Fourier transform' in our sense of a constant function on $\mathscr{O}_{\rho}$.

We now describe more explicitly the techniques employed to obtain these results.

In Section 1 we study a calculus of operators on a representation space $(V, \rho)$ with respect to a non-zero G-orbit $\mathscr{M}$. The main notion here is that of an effective orbit - one that has the property that for $T \in$ End $V, T=0$ if and only if $\langle T m, m\rangle=0$ for all $m \in \mathscr{M}$. Then we may associate to each $T$ its symbol $\sigma_{T}(m)=\langle T m, m\rangle$. We also define an orbit to be symmetric if

$$
\int_{\mathscr{M}}\left\langle v_{1}, m\right\rangle\left\langle v_{2}, m\right\rangle\left\langle m, v_{3}\right\rangle\left\langle m, v_{4}\right\rangle d m
$$

is a multiple of $\left\langle v_{1}, v_{3}\right\rangle\left\langle v_{2}, v_{4}\right\rangle+\left\langle v_{1}, v_{4}\right\rangle\left\langle v_{2}, v_{3}\right\rangle$. Various integral formulae for symbols on a symmetric orbit are proven, and we conclude in Proposition 1.17 that a symmetric orbit is effective. 
In Section 2 we introduce some ideas to describe algebras of functions on a manifold $\mathscr{M}$ which carry a Hilbert space structure. We employ an integral-type notation to deal with certain bilinear forms that arise and use it to define the notion of a triple-kernel of the algebra (with respect to the inner product). This is a function on $\mathscr{M} \times \mathscr{M} \times \mathscr{M}$ which encodes the algebra structure in a fashion similar to the way in which the kernel of an operator encodes the operator.

If $\mathscr{M}$ is an effective orbit, the space of symbols $W$ on $\mathscr{M}$ turns out to carry two natural Hilbert space structures and an algebra structure. In Section 3 we study the operators which relate these two inner products. For a symmetric orbit, we develop explicit formulae for the kernels of these operators. We also study the triple-kernels for $W$, the main result being Theorem 3.11, giving a formula for the triple-kernel with respect to one of the inner products for a general effective orbit.

In Section 4, we relate the previous discussion to orbits in $\mathfrak{g}^{*}$ by introducing the moment map of a representation $(V, \rho)$. Since $G$ acts on the projective space $P V$ of $V$ in a Hamiltonian way, there is a canonical map (the moment map) $\Phi^{\prime}: P V \rightarrow \mathfrak{g}^{*}$. Now if $\Omega$ is the unit sphere in $V$ and $\epsilon: \Omega \rightarrow P V$ the natural map, we define $\Phi=\Phi^{\prime} \circ \epsilon$ and call it the moment map of $\rho$. Our definition is actually more direct but equivalent. We study the image of $\Phi$ and prove various functorial properties of it. These results are analogues of the functorial relationships between representations and co-adjoint orbits first discovered by Kirillov [9] for nilpotent groups. We show that the extremal set of $\operatorname{Im} \Phi$ is a single G-orbit $\mathscr{O}_{\rho}$ and that $\Phi^{-1}\left(\mathscr{O}_{\rho}\right)=\mathscr{M}_{\rho}$ is a single G-orbit in $\Omega$. Furthermore $\Phi: \mathscr{M}_{\rho} \rightarrow \mathscr{O}_{\rho}$ is an $S^{1}$ bundle, which we call the canonical bundle of $\rho$ (Proposition 4.9). In Theorem 4.11 we show that $\mathscr{M}_{\rho}$ is an effective orbit.

The association of the orbit $\mathscr{O}_{\rho}$ to each $\rho \in \hat{G}$ is injective and is closely related to classical descriptions of $\hat{G}$. If we fix a choice of positive roots, then $\mathscr{O}_{\rho}$ and $\mathscr{M}_{\rho}$ can be considered to be the orbits of the highest weight and highest weight vector respectively. It also turns out that the canonical bundle of $\rho$ is the same as that constructed in geometric quantization - this fact was communicated to us in conversation by I. Frenkel, to whom we are grateful.

In Section 5 we use $\Phi$ to transfer structure from $\mathscr{M}_{\rho}$ to $\mathscr{O}_{\rho}$. We show how the canonical 2-form on $\mathscr{O}_{\rho}$ and the connection 1-form on $\mathscr{M}_{\rho}$ are related to the complex structure of $V$. We define the space of functions $A_{\rho}$ on $\mathscr{O}_{\rho}$.

In Section 6 the main definitions concerning the Fourier kernel $e$ and the Fourier transform $F$ are introduced. The main results here have already been described.

In Section 7 we turn in some detail to the case of $G=S U(2)$. Here the 
integral orbits are spheres in $\mathfrak{g}^{*}$ of radii $\ell=0,1 / 2,1,3 / 2, \ldots$ We compute the Fourier kernel $e$ of $G$ in Theorem 7.5 and discover that it is closely related to the Cayley transform for $G$. At this point we remark that in the thesis Wildberger [22] we have constructed a similar Fourier transform theory for nilpotent Lie groups using entirely different methods (see also Arnal and Cortet [2]). For the group of real upper triangular matrices we found that the Fourier kernel also involved the Cayley transform. This phenomenon has been investigated for a wide class of nilpotent groups in Howe, Ratcliff and Wildberger [7] where it is shown that the role of the Cayley transform is closely related to the theory of the oscillator representation of the symplectic group.

We also remark that our Fourier transform theory coincides in the case of $S U(2)$ with that introduced by Sherman [15] in his work on Fourier analysis on spheres (see also Helgason [6]). In Theorems 7.12 and 7.13 we give formulae for the triple-kernels of the $*$-products on the integral spheres. These are related in a surprising way to certain classical identities of spherical trigonometry, and shed new light on the $*$-products of Moreno and Ortega-Navarro [14] as well as on Sherman's work. More generally, our theory provides an explicit construction of a *-product on any integral co-adjoint orbit of a compact semisimple Lie group. It would be of interest to compare this with the general construction of *-products on symplectic manifolds studied by Lichnerowicz [13], Gutt [5] and others.

This paper is meant to be largely self-contained, the major requirement of the reader being a familiarity with elementary representation theory, for which references are Varadarajan [18] and Helgason [6].

This paper has existed in preprint form for some years now. A different version of our geometric Fourier transform construction has since appeared in Figueroa, Gracia-Bondía and Várilly [4] (see also Várilly and Gracia-Bondía [19], Arnal, Cahen and Gutt [1]). Additional work on the relationship between representations and moment maps has appeared in Wildberger [23, 24] and Arnal and Ludwig [3].

\section{Section 1}

Let $G$ be a compact Lie group and $(V, \rho)$ an irreducible unitary representation of $G$. The Hermitian form on $V$ will be denoted by $\langle$,$\rangle , and will be taken to be$ linear in the first variable and conjugate linear in the second. Let $\operatorname{dim} V=n$. Let $\mathscr{M}$ be a non-zero orbit in $V$, so that there exists $v \neq 0$ in $V$ with $\mathscr{M}=$ 
$\{g \cdot v \mid g \in G\}$. Here the use of $\rho$ is suppressed, so that $g \cdot v=\rho(g)(v)$. The orbit $\mathscr{M}$ carries a G-invariant measure.

LEMMA 1.1. The G-invariant measure $d m$ on $\mathscr{M}$ may be normalised so that for any $v \in V$,

$$
v=\int_{\mathscr{M}}\langle v, m\rangle m d m
$$

PROOF. Consider the operator $A \in$ End $V$ defined by

$$
A(v)=\int_{\mathscr{M}}\langle v, m\rangle m d m
$$

for $v \in V$. Then for any $g \in G$,

$$
\begin{aligned}
A(g \cdot v) & =\int_{\mathscr{M}}\langle g \cdot v, m\rangle m d m \\
& =\int_{\mathscr{M}}\left\langle v, g^{-1} \cdot m\right\rangle m d m \\
& =\int_{\mathscr{M}}\langle v, m\rangle g \cdot m d m \\
& =g \cdot A(v) .
\end{aligned}
$$

Thus $A$ commutes with all $g \in G$ and so by Schur's Lemma is a multiple of the identity. By an appropriate normalisation of $d m$ we may choose the multiple to be 1 .

Fix the measure $d m$ on $\mathscr{M}$ so that (1.1) holds. Introduce $L^{2}(\mathscr{M}, d m)$ with inner product $\langle,\rangle_{2}$. Thus for $\phi_{1}, \phi_{2} \in L^{2}(\mathscr{M}, d m)$,

$$
\left\langle\phi_{1}, \phi_{2}\right\rangle_{2}=\int_{\mathscr{M}} \phi_{1}(m) \overline{\phi_{2}(m)} d m .
$$

For $v \in V$, let $\tilde{v}$ be the function on $\mathscr{M}$ defined by $\tilde{v}(m)=\langle v, m\rangle$. Let $\tilde{V}$ be the space of all $\tilde{v}, v \in V$, and let ${ }^{\sim}: V \rightarrow \tilde{V}$ be the map that sends $v$ to $\tilde{v}$.

LEMMA 1.2. $~: V \rightarrow \tilde{V} \subset L^{2}(\mathscr{M}, d m)$ is an isometry. 
PROOF. Since $(V, \rho)$ is irreducible, $\tilde{v}=0$ if and only if $v=0$, so $\sim$ is an isomorphism. If $v_{1}, v_{2} \in V$, then by Lemma 1.1 ,

$$
\begin{aligned}
\left\langle v_{1}, v_{2}\right\rangle & =\int_{\mathscr{\mu}}\left\langle v_{1}, m\right\rangle\left\langle m, v_{2}\right\rangle d m \\
& =\left\langle\tilde{v}_{1}, \tilde{v}_{2}\right\rangle_{2}
\end{aligned}
$$

DEFINITION 1.3. For $T \in$ End $V$, define $K_{T}: \mathscr{M} \times \mathscr{M} \rightarrow \mathbb{C}$, the kernel of $T$ with respect to $\mathscr{M}$, by the formula

$$
K_{T}\left(m^{\prime}, m\right)=\left\langle T \cdot m^{\prime}, m\right\rangle
$$

for $m^{\prime}, m \in \mathscr{M}$.

LEMMA 1.4. For $T_{1}, T_{2} \in$ End $V$ and $m^{\prime \prime}, m \in \mathscr{M}$,

$$
K_{T_{2} T_{1}}\left(m^{\prime \prime}, m\right)=\int_{\mathscr{M}} K_{T_{1}}\left(m^{\prime \prime}, m^{\prime}\right) K_{T_{2}}\left(m^{\prime}, m\right) d m^{\prime} .
$$

PROOF. For $T \in$ End $V$, let $\tilde{T}$ be the integral operator on $L^{2}(\mathscr{M}, d m)$ with kernel $K_{T}$. That is, for $\phi \in L^{2}(\mathscr{M}, d m)$ and $m \in \mathscr{M}$,

$$
\tilde{T} \phi(m)=\int_{\mathscr{M}} K_{T}\left(m^{\prime}, m\right) \phi\left(m^{\prime}\right) d m^{\prime} .
$$

Then for $v \in V$ and $m \in \mathscr{M}$,

$$
\begin{aligned}
\tilde{T} \tilde{v}(m) & =\int_{\mathscr{M}}\left\langle T \cdot m^{\prime}, m\right\rangle\left\langle v, m^{\prime}\right\rangle d m^{\prime} \\
& =\langle T \cdot v, m\rangle \\
& =(T v)^{\sim}(m) .
\end{aligned}
$$

Here we have used Lemma 1.1. Now let

$$
\tilde{V}^{\perp}=\left\{\phi \in L^{2}(\mathscr{M}, d m) \mid\langle\phi, \tilde{v}\rangle_{2}=0 \quad \forall v \in V\right\} .
$$

Then for $\phi \in \tilde{V}^{\perp}$ and $m \in \mathscr{M}$, 


$$
\begin{aligned}
\tilde{T} \phi(m) & =\int_{\mathscr{M}}\left\langle T m^{\prime}, m\right\rangle \phi\left(m^{\prime}\right) d m^{\prime} \\
& =\int_{\mathscr{M}} \phi\left(m^{\prime}\right) \overline{\left(T^{*} m\right)^{\sim}\left(m^{\prime}\right)} d m^{\prime} \\
& =\left\langle\phi,\left(T^{*} m\right)^{\sim}\right\rangle_{2} \\
& =0 .
\end{aligned}
$$

Here $T^{*}$ is the adjoint of $T$. Now the facts that $\tilde{T} \tilde{v}=(T v)^{\sim}$ for all $v \in V$ and $\tilde{T} \phi=0$ for all $\phi \in \tilde{V}^{\perp}$ imply that the kernel of $T_{2} T_{1}$ with respect to $\mathscr{M}$ is just the kernel of the integral operator $\tilde{T}_{2} \tilde{T}_{1}$ on $L^{2}(\mathscr{M}, d m)$. But it is a standard fact that this is

$$
K_{T_{2} T_{1}}\left(m^{\prime \prime}, m\right)=\int_{\mathscr{N}} K_{T_{1}}\left(m^{\prime \prime}, m^{\prime}\right) K_{T_{2}}\left(m^{\prime}, m\right) d m^{\prime}
$$

PROPOSITION 1.5. For $T \in$ End $V$, tr $T=\int_{\mathscr{M}} K_{T}(m, m) d m$.

PROOF. It follows by the argument in the previous Lemma that $\operatorname{tr} T=\operatorname{tr} \tilde{T}$ since $L^{2}(\mathscr{M}, d m)=\tilde{V} \oplus \tilde{V}^{\perp}$. But tr $\tilde{T}=\int_{\mathscr{M}} K_{T}(m, m) d m$.

Let $|\mathscr{M}|$, the modulus of $\mathscr{M}$, be the number $\sqrt{\langle m, m\rangle}$ for any $m \in \mathscr{M}$.

COROLlARY 1.6.

$$
\int_{\mathscr{M}} d m=n / \mid \mathscr{M}^{2}
$$

ProOF. Apply Proposition 1.5 to the identity $I \in$ End $V$. Then

$$
\operatorname{tr} I=n=\int_{\mathscr{M}}\langle m, m\rangle d m=\mid \mathscr{M}^{2} \int_{\mathscr{M}} d m
$$

so the result follows.

COROLLARY 1.7. If $\mid \mathscr{M}=1$, then $\int_{\mathscr{M}} d m=n$.

DEFINITION 1.8. For $T \in$ End $V$, define $\sigma_{T}: \mathscr{M} \rightarrow \mathbb{C}$, the symbol of $T$ with respect to $\mathscr{M}$, by the formula

$$
\sigma_{T}(m)=K_{T}(m, m) .
$$

Also let $W$ be the space of all $\sigma_{T}, T \in$ End $V$, and let $\sigma:$ End $V \rightarrow W$ be the map that sends $T$ to $\sigma_{T}$. 
We are primarily interested in orbits $\mathscr{M}$ for which the map $\sigma$ can be used to establish a symbol calculus.

DEFINITION 1.9. An orbit $\mathscr{M} \subset V$ is called effective if the map $\sigma$ is an isomorphism from End $V$ to $W$.

EXAMPLE 1.10. Let $G=S U(n)$ and $(V, \rho)$ be the standard (or defining) representation of $G$. Thus $\operatorname{dim} V=n$, and the orbits of $G$ are simply spheres. Let $\mathscr{M}$ be the unit sphere. Then $\mathscr{M}$ is an effective orbit. This is a restatement of the fact from elementary linear algebra that if $T \in$ End $V$ and $\langle T m, m\rangle=0$ for all $m \in \mathscr{M}$, then $T=0$. This fact follows from the formula

$$
\begin{aligned}
& \langle T v, w\rangle=\frac{1}{4}(\langle T(v+w), v+w\rangle-\langle T(v-w), v-w\rangle \\
& +i\langle T(v+i w), v+i w\rangle-i\langle T(v-i w), v-i w\rangle)
\end{aligned}
$$

for all $v, w \in v$. For $v_{1}, v_{2}, v_{3}, v_{4} \in v$ define

$$
P_{\mathscr{M}}\left(v_{1}, v_{2}, v_{3}, v_{4}\right)=\int_{\mathscr{M}}\left\langle v_{1}, m\right\rangle\left\langle v_{2}, m\right\rangle\left\langle m, v_{3}\right\rangle\left\langle m, v_{4}\right\rangle d m .
$$

DEFINITION 1.11. An orbit $\mathscr{M} \subset V$ is called symmetric if there exists a constant $c=c(\mathscr{M})$ such that for all $v_{1}, v_{2}, v_{3}, v_{4} \in V$,

$$
P_{\mathscr{M}}\left(v_{1}, v_{2}, v_{3}, v_{4}\right)=c\left(\left\langle v_{1}, v_{3}\right\rangle\left\langle v_{2}, v_{4}\right\rangle+\left\langle v_{1}, v_{4}\right\rangle\left\langle v_{2}, v_{3}\right\rangle\right)
$$

PROPOSITION 1.12. Let $\mathscr{M} \subset V$ be the unit sphere, considered as an orbit of $S U(n)$ acting on $V$. Then $\mathscr{M}$ is symmetric and for $v_{1}, v_{2}, v_{3}, v_{4} \in V$,

$$
P_{\mathscr{M}}\left(v_{1}, v_{2}, v_{3}, v_{4}\right)=(1 /(n+1))\left(\left\langle v_{1}, v_{3}\right\rangle\left\langle v_{2}, v_{4}\right\rangle+\left\langle v_{1}, v_{4}\right\rangle\left\langle v_{2}, v_{3}\right\rangle\right)
$$

PROOF. By multilinearity if suffices to prove the result for $v_{1}, v_{2}, v_{3}, v_{4}$ members of an orthonormal basis of $V$. This is then an exercise in advanced calculus.

LEMMA 1.13. Let $\mathscr{M} \subset V$ be a symmetric orbit with constant $c=c(\mathscr{M})$. Then for $T_{1}, T_{2} \in$ End $V$,

$$
\int_{\mathscr{M}}\left\langle T_{1} m, m\right\rangle\left\langle T_{2} m, m\right\rangle d m=c\left(\operatorname{tr} T_{1} \operatorname{tr} T_{2}+\operatorname{tr} T_{1} T_{2}\right) .
$$


PROOF. Fix an orthonormal basis $\left\{v_{1}, \ldots, v_{n}\right\}$ of $V$. For $1 \leq i, j \leq n$ define $T_{i j} \in$ End $V$ by the equation $T_{i j}(v)=\left\langle v, v_{j}\right\rangle v_{i}$ for $v \in V$. These form a basis of End $V$ so we may find constants $\alpha_{i j}, \beta_{i j}$ such that $T_{1}=\sum_{i, j} \alpha_{i j} T_{i j}$ and $T_{2}=\sum_{i, j} \beta_{i j} T_{i j}$. Then

$$
\begin{aligned}
\int_{\mathscr{M}}\left\langle T_{1} m, m\right\rangle\left\langle T_{2} m, m\right\rangle d m & =\sum_{i, j} \sum_{k, l} \alpha_{i j} \beta_{k l} \int_{\mathscr{M}}\left\langle T_{i j} m, m\right\rangle\left\langle T_{k l} m, m\right\rangle d m \\
& =\sum_{i, j} \sum_{k, l} \alpha_{i j} \beta_{k l} P_{\mathscr{M}}\left(v_{i}, v_{k}, v_{j}, v_{l}\right) \\
& =c \sum_{i, j} \sum_{k, l} \alpha_{i j} \beta_{k l}\left(\left\langle v_{i}, v_{j}\right\rangle\left\langle v_{k}, v_{l}\right\rangle+\left\langle v_{i}, v_{l}\right\rangle\left\langle v_{k}, v_{j}\right\rangle\right) \\
& =c \sum_{i, j} \sum_{k, l} \alpha_{i j} \beta_{k l}\left(\delta_{i j} \delta_{k l}+\delta_{i l} \delta_{k j}\right) \\
& =c\left(\sum_{i=1}^{n} \alpha_{i i} \sum_{k=1}^{n} \beta_{k k}+\sum_{i, j} \alpha_{i j} \beta_{j i}\right) \\
& =c\left(\operatorname{tr} T_{1} \operatorname{tr} T_{2}+\operatorname{tr} T_{1} T_{2}\right) .
\end{aligned}
$$

COROLLARY 1.14 .

$$
c=c(\mathscr{M})=\mid \mathscr{M}^{2} /(n+1)
$$

PROOF. Let $T_{1}=T_{2}=I$ in the previous lemma. Then

$$
\int_{\mathscr{M}}\langle m, m\rangle^{2} d m=c\left(n^{2}+n\right) .
$$

But from Corollary 1.6,

$$
\int_{\mathscr{M}}\langle m, m\rangle^{2} d m=|\mathscr{M}|^{4} \int_{\mathscr{M}} d m=\mid \mathscr{M}^{2} n
$$

so that $c=|\mathscr{M}|^{2} /(n+1)$

COROLLARY 1.15. If $|\mathscr{M}|=1$ then $c=c(\mathscr{M})=1 /(n+1)$.

The constant $c$ is thus independent of the symmetric orbit $\mathscr{M}$ if $|\mathscr{M}|=1$. 
PROPOSITION 1.16. Let $\mathscr{M} \subset V$ be a symmetric orbit. Then for $T \in$ End $V$ and $v, w \in V$,

$$
\langle T v, w\rangle=\int_{\mathscr{M}}\langle T m, m\rangle\left(\frac{(n+1)}{\mid \mathscr{M}^{2}}\langle v, m\rangle\langle m, w\rangle-\langle v, w\rangle\right) d m .
$$

PROOF. Write $T=\sum_{i, j} \alpha_{i j} T_{i j}$ in the notation of the proof of Lemma 1.13. Then for $v, w \in V$,

$$
\begin{aligned}
\int_{\mathscr{M}}\langle T m, m\rangle\langle v, m\rangle\langle m, w\rangle d m & =\sum_{i, j} \alpha_{i j} P_{\mathscr{M}}\left(v_{i}, v, v_{j}, w\right) \\
& =\frac{\mid \mathscr{M}^{2}}{n+1} \sum_{i, j} \alpha_{i j}\left(\left\langle v_{i}, v_{j}\right\rangle\langle v, w\rangle+\left\langle v_{i}, w\right\rangle\left\langle v, v_{j}\right\rangle\right) \\
& =\frac{\mid \mathscr{M}^{2}}{n+1}\left(\langle v, w\rangle \sum_{i=1}^{n} \alpha_{i i}+\sum_{i, j} \alpha_{i j}\left\langle T_{i j} v, w\right\rangle\right) \\
& =\frac{\mid \mathscr{M}^{2}}{n+1}(\langle v, w\rangle \operatorname{tr} T+\langle T v, w\rangle) .
\end{aligned}
$$

Now since tr $T=\int_{\mathscr{M}} K_{T}(m, m) d m=\int_{\mathscr{M}}\langle T m, m\rangle d m$ (from Proposition 1.5), the result follows.

Proposition 1.16 shows how to recover the values $\langle T v, w\rangle(v, w \in V)$ from the symbol $\sigma_{T}$ of $T$. In particular, we have the following immediate consequence.

PROPOSITION 1.17. If $\mathscr{M} \subset V$ is a symmetric orbit, then it is also an effective orbit.

We may also write down an integral alternative to the formula of Example 1.10 .

PROPOSITION 1.18. Let $\mathscr{M} \subset V$ be the unit sphere. Let $T \in$ End $V$ and $\sigma_{T}$ be its symbol with respect to $\mathscr{M}$. Then for $v, w \in V$,

$$
\langle T v, w\rangle=\int_{\mathscr{M}} \sigma_{T}(m)((n+1)\langle v, m\rangle\langle m, w\rangle-\langle v, w\rangle) d m .
$$




\section{Section 2}

Let $\mathscr{M}$ be a compact manifold and $W$ a finite-dimensional space of continuous functions on $\mathscr{M}$, closed under conjugation and containing the constants. Suppose further that $W$ is a Hilbert space with inner product $\langle,\rangle_{\alpha}$. In the application we have in mind, $\alpha$ will be an index for several Hilbert space structures on $W$.

For a positive integer $k$, define $W^{(k)}$ to be the space of all functions on $\mathscr{M} \times \ldots \times \mathscr{M}(k$ factors $)$ which belong to $W$ in each argument. Consider the symmetric bilinear form on $W$ defined by $\left(w_{1}, w_{2}\right)_{\alpha}=\left\langle w_{1}, \bar{w}_{2}\right\rangle_{\alpha}$ for $w_{1}, w_{2} \in$ $W$. We introduce the integral-like symbol $\int_{\alpha} d m$ for this form and write

$$
\left(w_{1}, w_{2}\right)_{\alpha}=\int_{\alpha} w_{1}(m) w_{2}(m) d m
$$

The reader is warned that this symbol does not have its standard meaning here, but nevertheless behaves in a fashion similar to the usual integral under elementary operations. The following facts are immediate.

LEMMA 2.1. For any $w_{1}, w_{2}, w_{3} \in W$ and $c \in \mathbb{C}$,

(a) $\int_{\alpha} w_{1}(m) w_{2}(m) d m=\int_{\alpha} w_{2}(m) w_{1}(m) d m ;$

(b) $\int_{\alpha}\left(c w_{1}\right)(m) w_{2}(m) d m=\int_{\alpha} w_{1}(m)\left(c w_{2}\right)(m) d m=c \int_{\alpha} w_{1}(m) w_{2}(m) d m$;

(c) $\int_{\alpha} w_{1}(m)\left(w_{2}+w_{3}\right)(m) d m=\int_{\alpha} w_{1}(m) w_{2}(m) d m+\int_{\alpha} w_{1}(m) w_{3}(m) d m ;$

(d) $\int_{\alpha} \bar{w}_{1}(m) \bar{w}_{2}(m) d m=\overline{\int_{\alpha} w_{1}(m) w_{2}(m) d m}$.

Now suppose that $\phi_{1} \in W^{(k)}$ and $\phi_{2} \in W^{(\ell)}$ with $\phi_{1}=\phi_{1}\left(m_{11}, \ldots, m_{1 k}\right)$ and $\phi_{2}=\phi_{2}\left(m_{21}, \ldots, m_{2 \ell}\right)$. Let $1 \leq i \leq k, 1 \leq j \leq \ell$. Then we can extend the above notation to give meaning to the expression

$$
\begin{aligned}
\int_{\alpha} \phi_{1}\left(m_{11}, \ldots, m_{1, i-1}, m, m_{1, i+1}, \ldots, m_{1 k}\right) \\
\cdot \phi_{2}\left(m_{21}, \ldots, m_{2, j-1}, m, m_{2, j+1}, \ldots, m_{2 \ell}\right) d m .
\end{aligned}
$$


The result is an element of $W^{k+\ell-2}$ in the variables $m_{11}, \ldots, \hat{m}_{1 i}, \ldots, m_{1 k}$, $m_{21}, \ldots, \hat{m}_{2 j}, \ldots, m_{2 \ell}$, where ${ }^{\wedge}$ means deletion. More generally we may 'integrate' in this fashion any finite product of functions belonging to $W^{(\infty)}=$ $\bigoplus_{k=1}^{\infty} W^{(k)}$ as long as the variable of integration appears as an argument of exactly two of them. We also consider 'multiple integrals', that is, expressions such as

$$
\int_{\alpha}\left(\int_{\alpha} \phi_{1}\left(m_{1}, m_{2}\right) \phi_{2}\left(m_{1}, m_{2}\right) d m_{1}\right) d m_{2}=\int_{\alpha} \int_{\alpha} \phi_{1}\left(m_{1}, m_{2}\right) \phi_{2}\left(m_{1}, m_{2}\right) d m_{1} d m_{2} .
$$

We leave the reader to check that we may freely interchange the order of integration in any such multiple integral. Thus the quantity in the above expression is also equal to

$$
\int_{\alpha}\left(\int_{\alpha} \phi_{1}\left(m_{1}, m_{2}\right) \phi_{2}\left(m_{1}, m_{2}\right) d m_{2}\right) d m_{1}
$$

Such manipulations will be made without further comment in what follows. We now show how to use the above conventions to introduce some familiar objects associated to $W$ and End $W$.

DEFINITION 2.2. A reproducing kernel for $W$ with respect to $\langle,\rangle_{\alpha}$ is a function $R_{\alpha} \in W^{(2)}$ such that for all $w \in W$,

$$
w(m)=\int_{\alpha} R_{\alpha}\left(m^{\prime}, m\right) w\left(m^{\prime}\right) d m^{\prime} .
$$

DEFINITION 2.3. If $T \in$ End $W$, a kernel for $T$ with respect to $\langle,\rangle_{\alpha}$ is a function $K_{\alpha}(T) \in W^{(2)}$ such that for all $w \in W$,

$$
T w(m)=\int_{\alpha} K_{\alpha}(T)\left(m^{\prime}, m\right) w\left(m^{\prime}\right) d m^{\prime} .
$$

Note that a reproducing kernel for $W$ is just a kernel for the identity $I \in$ End $W$. (When $\alpha$ is fixed or there is no possibility of confusion, we will drop the reference to $\langle,\rangle_{\alpha}$.)

The following is a standard fact whose proof we leave to the reader.

LEMMA 2.4. Suppose $\operatorname{dim} W=p$ and that $\left\{e_{1}, \ldots, e_{p}\right\}$ is an orthonormal basis of $W$ with respect to $\langle,\rangle_{\alpha}$. Then for any $T \in \mathrm{End} W$, a kernel for $T$ with respect to $\langle,\rangle_{\alpha}$ is

$$
K_{\alpha}(T)\left(m^{\prime}, m\right)=\sum_{i=1}^{p} \overline{e_{i}\left(m^{\prime}\right)} T e_{i}(m)
$$


Furthermore it is unique (so does not depend on the particular basis). In particular

$$
R_{\alpha}\left(m^{\prime}, m\right)=\sum_{i=1}^{p} \overline{e_{i}\left(m^{\prime}\right)} e_{i}(m)
$$

is the unique reproducing kernel for $W$ with respect to $\langle,\rangle_{\alpha}$.

Now suppose that the space $W$ has in addition an algebra structure, denoted by $x$. It will be useful to construct an object which will encode the algebra structure of $(W, \times)$ in a fashion similar to the way the kernel $K_{\alpha}(T)$ encodes the operator $T \in$ End $W$.

DEFINITION 2.5. A triple-kernel (or 3-kernel) for the algebra $(W, \times)$ with respect to $\langle,\rangle_{\alpha}$ is a function $B_{\alpha} \in W^{(3)}$ such that for all $w_{1}, w_{2} \in W$

$$
w_{1} \times w_{2}(m)=\int_{\alpha} \int_{\alpha} B_{\alpha}\left(m, m_{1}, m_{2}\right) w_{1}\left(m_{1}\right) w_{2}\left(m_{2}\right) d m_{1} d m_{2} .
$$

LEMMA 2.6. Suppose as before that $\left\{e_{1}, \ldots, e_{p}\right\}$ is an orthonormal basis of $W$ with respect to $\langle,\rangle_{\alpha}$ and that for $1 \leq i, j \leq p$,

$$
e_{i} \times e_{j}=\sum_{k=1}^{p} c_{i j}^{k} e_{k}, \quad c_{i j}^{k} \in \mathbb{C} .
$$

Then a triple-kernel for the algebra $(W, \times)$ with respect to $\langle,\rangle_{\alpha}$ is given by

$$
B_{\alpha}\left(m, m_{1}, m_{2}\right)=\sum_{i, j, k} c_{i j}^{k} \overline{e_{i}\left(m_{1}\right) e_{j}\left(m_{2}\right)} e_{k}(m) .
$$

Furthermore it is unique (so does not depend on the choice of basis).

PROOF. Let $w_{1}, w_{2} \in W$ with $w_{1}=\sum_{i=1}^{p} a_{i} e_{i}, w_{2}=\sum_{i=1}^{p} b_{i} e_{i}$. Then

$$
\begin{aligned}
\int_{\alpha} \int_{\alpha} \sum_{i, j, k} c_{i j}^{k} & \overline{e_{i}\left(m_{1}\right) e_{j}\left(m_{2}\right)} e_{k}(m) w_{1}\left(m_{1}\right) w_{2}\left(m_{2}\right) d m_{1} d m_{2} \\
= & \sum_{i, j, k} \sum_{r=1}^{p} \sum_{s=1}^{p} c_{i j}^{k} a_{r} b_{s} \int_{\alpha} \int_{\alpha} \overline{e_{i}\left(m_{1}\right) e_{j}\left(m_{2}\right)} e_{r}\left(m_{1}\right) e_{s}\left(m_{2}\right) e_{k}(m) d m_{1} d m_{2} \\
& =\sum_{i, j, k} \sum_{r, s} c_{i j}^{k} a_{r} b_{s} \delta_{r i} \delta_{s j} e_{k}(m)
\end{aligned}
$$




$$
\begin{aligned}
& =\sum_{r, s} a_{r} b_{s} \sum_{k} c_{r s}^{k} e_{k}(m) \\
& =\sum_{r, s} a_{r} b_{s} e_{r} \times e_{s}(m) \\
& =w_{1} \times w_{2}(m) .
\end{aligned}
$$

Thus $B_{\alpha}\left(m, m_{1}, m_{2}\right)=\sum_{i, j, k} c_{i j}^{k} \overline{e_{i}\left(m_{1}\right) e_{j}\left(m_{2}\right)} e_{k}(m)$ is a triple-kernel for the algebra $(W, \times)$ with respect to $\langle,\rangle_{\alpha}$. Its uniqueness follows directly from the condition that $B_{\alpha} \in W^{(3)}$.

The next few results show how properties of the algebra $(W, \times)$ are reflected in properties of the triple-kernel $B_{\alpha}$.

PROPOSITION 2.7. $(W, x)$ is associative if and only if for all $m_{1}, m_{2}, m_{3}, m_{4}$ $\in \mathscr{M}$

$$
\int_{\alpha} B_{\alpha}\left(m, m_{1}, m_{2}\right) B_{\alpha}\left(m_{4}, m, m_{3}\right) d m=\int_{\alpha} B_{\alpha}\left(m_{4}, m_{1}, m\right) B_{\alpha}\left(m, m_{2}, m_{3}\right) d m
$$

ProOF. Let $w_{1}, w_{2}, w_{3} \in W$. Then

$$
\begin{aligned}
& \left(w_{1} \times w_{2}\right) \times w_{3}\left(m_{4}\right) \\
& =\int_{\alpha} \int_{\alpha} B_{\alpha}\left(m_{4}, m, m_{3}\right) \iint_{\alpha} B_{\alpha}\left(m, m_{1}, m_{2}\right) w_{1}\left(m_{1}\right) w_{2}\left(m_{2}\right) d m_{1} d m_{2} w_{3}\left(m_{3}\right) d m d m_{3}
\end{aligned}
$$

while

$$
\begin{aligned}
& w_{1} \times\left(w_{2} \times w_{3}\right)\left(m_{4}\right) \\
& =\int_{\alpha} \int_{\alpha} B_{\alpha}\left(m_{4}, m_{1}, m\right) w_{1}\left(m_{1}\right) \iint_{\alpha} B_{\alpha}\left(m, m_{2}, m_{3}\right) w_{2}\left(m_{2}\right) w_{3}\left(m_{3}\right) d m_{2} d m_{3} d m_{1} d m .
\end{aligned}
$$

Thus $(W, \times)$ is associative if and only if for all $m_{4} \in \mathscr{M}$ and for all $w_{1}, w_{2}, w_{3} \in$ $W$,

$$
\int_{\alpha} \int_{\alpha} \int_{\alpha} C_{\alpha}\left(m_{1}, m_{2}, m_{3}, m_{4}\right) w_{1}\left(m_{1}\right) w_{2}\left(m_{2}\right) w_{3}\left(m_{3}\right) d m_{1} d m_{2} d m_{3}=0
$$

where 


$$
\begin{aligned}
& C_{\alpha}\left(m_{1}, m_{2}, m_{3}, m_{4}\right) \\
& \quad=\int_{\alpha} B_{\alpha}\left(m_{4}, m, m_{3}\right) B_{\alpha}\left(m, m_{1}, m_{2}\right)-B_{\alpha}\left(m_{4}, m_{1}, m\right) B_{\alpha}\left(m, m_{2}, m_{3}\right) d m .
\end{aligned}
$$

But since $B_{\alpha} \in W^{(3)}$, it is clear that $C_{\alpha} \in W^{(4)}$. Thus $C_{\alpha}$ must be identically zero, and we are done.

PROPOSITION 2.8. $(W, \times)$ is commutative if and only if

$$
B_{\alpha}\left(m, m_{1}, m_{2}\right)=B_{\alpha}\left(m, m_{2}, m_{1}\right)
$$

PROOF. Immediate from the definitions and a change of variable.

PROPOSITION 2.9. $1 \in W$ is the identity of $(W, x)$ if and only if both

$$
\begin{aligned}
& \int_{\alpha} B_{\alpha}\left(m, m_{1}, m_{2}\right) d m_{2}=R_{\alpha}\left(m_{1}, m\right) \quad \text { and } \\
& \int_{\alpha} B_{\alpha}\left(m, m_{1}, m_{2}\right) d m_{1}=R_{\alpha}\left(m_{2}, m\right) .
\end{aligned}
$$

PROOF. This follows immediately from the fact that

$$
\begin{aligned}
& w \times 1(m)=\int_{\alpha} \int_{\alpha} B_{\alpha}\left(m, m_{1}, m_{2}\right) w\left(m_{1}\right) d m_{1} d m_{2} \quad \text { and } \\
& 1 \times w(m)=\int_{\alpha} \int_{\alpha} B_{\alpha}\left(m, m_{1}, m_{2}\right) w\left(m_{2}\right) d m_{1} d m_{2}
\end{aligned}
$$

since of course $1(m)=1$.

PROPOSITION 2.10. The algebra $(W, \times)$ has the property that $\overline{w_{1} \times w_{2}}=$ $\bar{w}_{2} \times \bar{w}_{1}$ if and only if

$$
B_{\alpha}\left(m, m_{2}, m_{1}\right)=\overline{B_{\alpha}\left(m, m_{1}, m_{2}\right)} .
$$

PROOF. Left to the reader. 
PROPOSITION 2.11. The algebra $(W, \times)$ has the property that for all $w_{1}, w_{2} \in$ $W$

$$
\int_{\alpha} w_{1} \times \bar{w}_{2}(m) d m=\left\langle w_{1}, w_{2}\right\rangle_{\alpha}
$$

if and only if

$$
\int_{\alpha} B_{\alpha}\left(m, m_{1}, m_{2}\right) d m=R_{\alpha}\left(m_{2}, m_{1}\right)
$$

PROOF. We first remark that the left hand side of both expressions is valid since $1 \in W$. For $w_{1}, w_{2} \in W$,

$$
\int_{\alpha} w_{1} \times \bar{w}_{2}(m) d m=\int_{\alpha} \int_{\alpha} \int_{\alpha} B_{\alpha}\left(m, m_{1}, m_{2}\right) w_{1}\left(m_{1}\right) \bar{w}_{2}\left(m_{2}\right) d m_{1} d m_{2} d m
$$

while

$$
\left\langle w_{1}, w_{2}\right\rangle_{\alpha}=\int_{\alpha} w_{1}\left(m_{2}\right) \bar{w}_{2}\left(m_{2}\right) d m_{2} .
$$

Thus $(W, \times)$ has the required property if and only if for all $w_{1} \in W$,

$$
w_{1}\left(m_{2}\right)=\int_{\alpha} \int_{\alpha} B_{\alpha}\left(m, m_{1}, m_{2}\right) d m w_{1}\left(m_{1}\right) d m_{1},
$$

that is, if and only if

$$
\int_{\alpha} B_{\alpha}\left(m, m_{1}, m_{2}\right) d m=R_{\alpha}\left(m_{1}, m_{2}\right)
$$

These propositions show that in general an asymmetry exists between the first argument of a triple-kernel $B_{\alpha}\left(m, m_{1}, m_{2}\right)$ and the last two. We will say that a triple-kernel $B_{\alpha}$ is symmetric if

$$
B_{\alpha}\left(m, m_{1}, m_{2}\right)=B_{\alpha}\left(m_{1}, m_{2}, m\right)=B_{\alpha}\left(m_{2}, m, m_{1}\right)
$$

for all $m, m_{1}, m_{2} \in \mathscr{M}$. An algebra $(W, \mathrm{x})$ will be called symmetric with respect to $\langle,\rangle_{\alpha}$ if its triple-kernel $B_{\alpha}$ is symmetric.

PROPOSITION 2.12. The algebra $(W, \times)$ is symmetric with respect to $\langle,\rangle_{\alpha}$ if and only if

$$
\left\langle w_{1} \times w_{2}, \bar{w}_{3}\right\rangle_{\alpha}=\left\langle w_{2} \times w_{3}, \bar{w}_{1}\right\rangle_{\alpha}=\left\langle w_{3} \times w_{1}, \bar{w}_{2}\right\rangle_{\alpha}
$$

for all $w_{1}, w_{2}, w_{3} \in W$.

PROOF. This follows along the same lines as the previous arguments. 


\section{Section 3}

The definitions and notation of Section 2 will now be applied to the situation in Section 1. Thus $\mathscr{M} \subset V$ will be taken to be an effective orbit of the compact group $G$, and to each $T \in$ End $V$ we have the unique symbol $\sigma_{T}$. The space $W$ of all symbols on $\mathscr{M}$ is a finite dimensional space of continuous functions on $\mathscr{M}$ which contains the constants and is closed under conjugation. Let $\langle,\rangle_{2}$ be the inner product on $W$ which is the restriction of the inner product from $L^{2}(\mathscr{M}, d m)$, so that for $w_{1}, w_{2} \in W$,

$$
\left\langle w_{1}, w_{2}\right\rangle_{2}=\int_{\mathscr{M}} w_{1}(m) \overline{w_{2}(m)} d m .
$$

We may also consider another inner product on $W$, arising from the Hilbert space structure of End $V$.

DEFINITION 3.1. For $w_{1}, w_{2} \in W$ with $\sigma^{-1}\left(w_{1}\right)=T_{1}, \sigma^{-1}\left(w_{2}\right)=T_{2}$, define

$$
\left\langle w_{1}, w_{2}\right\rangle_{1}=\operatorname{tr}\left(T_{1} T_{2}^{*}\right) .
$$

The two Hilbert space structures $\langle,\rangle_{1}$ and $\langle,\rangle_{2}$ are in general not the same. Let $\tilde{\eta}: W \rightarrow W$ be the unique invertible operator such that

$$
\left\langle\tilde{\eta}\left(w_{1}\right), w_{2}\right\rangle_{1}=\left\langle w_{1}, w_{2}\right\rangle_{2}
$$

for all $w_{1}, w_{2} \in W$. Equivalently consider the operator $\eta:$ End $V \rightarrow$ End $V$ such that $\sigma_{\eta(T)}=\tilde{\eta}\left(\sigma_{T}\right)$ for all $T \in$ End $V$.

Proposition 3.2. For $T \in$ End $V$ and $v \in V$,

$$
\eta(T)(v)=\int_{\mathscr{M}} \sigma_{T}(m)\langle v, m\rangle m d m .
$$

PROOF. Let $\mu:$ End $V \rightarrow$ End $V$ be defined by

$$
\mu(T)(v)=\int_{\mathscr{M}} \sigma_{T}(m)\langle v, m\rangle m d m
$$

for $T \in$ End $V$ and $v \in V$. The kernel of $\mu(T)$ with respect to $\mathscr{M}$ is

$$
K_{\mu(T)}\left(m_{1}, m_{2}\right)=\int_{\mathscr{M}} \sigma_{T}(m)\left\langle m_{1}, m\right\rangle\left\langle m, m_{2}\right\rangle m d m .
$$


Thus for $T_{1}, T_{2} \in$ End $V, \mu\left(T_{1}\right) T_{2}^{*}$ has kernel

$$
\begin{aligned}
K_{\mu\left(T_{1}\right) T_{2}^{*}}\left(m_{1}, m_{2}\right) & =\int_{\mathscr{K}} K_{T_{2}^{*}}\left(m_{1}, m\right) K_{\mu\left(T_{1}\right)}\left(m, m_{2}\right) d m \\
& =\int_{\mathscr{A}} \int_{\mathscr{K}}\left\langle m_{1}, T_{2} m\right\rangle \sigma_{T_{1}}\left(m^{\prime}\right)\left\langle m, m^{\prime}\right\rangle\left\langle m^{\prime}, m_{2}\right\rangle d m^{\prime} d m \\
& =\int_{\mathscr{K}} \sigma_{T_{1}}\left(m^{\prime}\right)\left\langle m_{1}, T_{2} m^{\prime}\right\rangle\left\langle m^{\prime}, m_{2}\right\rangle d m^{\prime}
\end{aligned}
$$

from Lemma 1.4.

Thus by Proposition 1.5,

$$
\begin{aligned}
\operatorname{tr} \mu\left(T_{1}\right) T_{2}^{*} & =\int_{\mathscr{M}} \int_{\mathscr{\mathscr { K }}} \sigma_{T_{1}}\left(m^{\prime}\right)\left\langle m_{1}, T_{2} m^{\prime}\right\rangle\left\langle m^{\prime}, m_{1}\right\rangle d m^{\prime} d m_{1} \\
& =\int_{\mathscr{M}} \sigma_{T_{1}}\left(m^{\prime}\right)\left\langle m^{\prime}, T_{2} m^{\prime}\right\rangle d m^{\prime} \\
& =\int_{\mathscr{M}} \sigma_{T_{1}}\left(m^{\prime}\right) \overline{\sigma_{T_{2}}\left(m^{\prime}\right)} d m^{\prime} \\
& =\left\langle\sigma_{T_{1}}, \sigma_{T_{2}}\right\rangle_{2} .
\end{aligned}
$$

\section{COROLlaRY 3.3.}

(a) $\quad$ For $T \in$ End $V, \quad \operatorname{tr} \eta(T)=|\mathscr{M}|^{2} \operatorname{tr} T$

(b) $\quad$ For $I=$ Identity, $\quad \eta I=\mid \mathscr{M}^{2} I$.

PROOF.

(a)

$$
\begin{aligned}
\operatorname{tr} \eta(T) & =\int_{\mathscr{H}} K_{\eta(T)}(m, m) d m \\
& =\int_{\mathscr{M}} \int_{\mathscr{M}} \sigma_{T}\left(m^{\prime}\right)\left\langle m, m^{\prime}\right\rangle\left\langle m^{\prime}, m\right\rangle d m^{\prime} d m \\
& =\int_{\mathscr{M}} \sigma_{T}\left(m^{\prime}\right)\left\langle m^{\prime}, m^{\prime}\right\rangle d m^{\prime} \\
& =|\mathscr{M}|^{2} \operatorname{tr} T
\end{aligned}
$$

(b)

$$
\begin{aligned}
\eta(I)(v) & =\int_{\mathscr{M}}\langle m, m\rangle\langle v, m\rangle m d m \\
& =\mid \mathscr{M}^{2} v .
\end{aligned}
$$


LEMMA 3.4. $\eta$ commutes with the action of $G$ on End $V$ by conjugation.

Proof. For $g \in G, v \in V$ and $T \in$ End $V$ we use Proposition 3.2 to obtain

$$
\begin{aligned}
\eta(T)(g \cdot v) & =\int_{\mathscr{M}} \sigma_{T}(m)\langle g \cdot v, m\rangle m d m \\
& =\int_{\mathscr{M}} \sigma_{T}(g \cdot m)\langle v, m\rangle g \cdot m d m
\end{aligned}
$$

But $\sigma_{T}(g \cdot m)=\left\langle g^{-1} \cdot T \cdot g \cdot m, m\right\rangle$ so

$$
g^{-1} \cdot(\eta(T)(g \cdot v))=\eta\left(g^{-1} T g\right)(v) .
$$

PROPOSITION 3.5. The kernel for $\tilde{\eta}: W \rightarrow W$ with respect to $\langle,\rangle_{2}$ is the function $N \in W^{(2)}$ given by

$$
N\left(m^{\prime}, m\right)=\left|\left\langle m^{\prime}, m\right\rangle\right|^{2} .
$$

PROOF. We must show that $N\left(m^{\prime}, m\right) \in W^{(2)}$ and that for all $w \in W$ and $m \in \mathscr{M}$,

$$
\tilde{\eta}(w)(m)=\int_{\mathscr{M}} N\left(m^{\prime}, m\right) w\left(m^{\prime}\right) d m .
$$

Now for fixed $m^{\prime} \in \mathscr{M}$ the operator $T_{m^{\prime}}, \in$ End $V$ defined by $T_{m^{\prime}}(v)=\left\langle v, m^{\prime}\right\rangle m^{\prime}$ has symbol $\sigma_{T_{m^{\prime}}}(m)=\left|\left\langle m^{\prime}, m\right\rangle\right|^{2}$ so by the symmetry of $N, N \in W^{(2)}$.

Now let $w \in W$ and suppose that $w=\sigma_{T}, T \in$ End $V$. For $m \in \mathscr{M}$, use Proposition 3.2 to obtain

$$
\begin{aligned}
\tilde{\eta}(w)(m) & =\sigma_{\eta(T)}(m) \\
& =\langle\eta(T) m, m\rangle \\
& =\int_{\mathscr{M}} \sigma_{T}\left(m^{\prime}\right)\left\langle m, m^{\prime}\right\rangle\left\langle m^{\prime}, m\right\rangle d m^{\prime} \\
& =\int_{\mathscr{M}} N\left(m^{\prime}, m\right) w\left(m^{\prime}\right) d m^{\prime}
\end{aligned}
$$


It now follows that knowledge of $\eta^{-1}$ (or equivalently, $\tilde{\eta}^{-1}$ ) allows one to recover an operator $T \in$ End $V$ from its symbol $\sigma_{T}$. In fact, from Proposition 3.2 it follows that for $v, w \in V$,

$$
\langle T v, w\rangle=\int_{\mathscr{M}} \sigma_{\eta^{-1}(T)}(m)\langle v, m\rangle\langle m, w\rangle d m .
$$

It is thus an interesting problem to write down explicitly the kernel of $\tilde{\eta}^{-1}$. In the case of $\mathscr{M}$ a symmetric orbit we use the results of Section 1 to do this. For convenience we treat only the case $|\mathscr{M}|=1$.

PROPOSITION 3.6. Let $\mathscr{M}$ be a symmetric orbit with $|\mathscr{M}|=1$. Then the kernel of $\tilde{\eta}^{-1}: W \rightarrow W$ with respect to $\langle,\rangle_{2}$ is the function $M \in W^{(2)}$ given by

$$
M\left(m^{\prime}, m\right)=(n+1)^{2}\left|\left\langle m^{\prime}, m\right\rangle\right|^{2}-(n+2) .
$$

PROOF. It is clear that $M \in W^{(2)}$. Thus let $\tilde{\zeta}: W \rightarrow W$ be the operator whose kernel with respect to $\langle,\rangle_{2}$ is $M\left(m^{\prime}, m\right)$. Then the kernel of $\tilde{\zeta} \tilde{\eta}$ is by Lemma 1.4

$$
\begin{aligned}
K\left(m^{\prime \prime}, m\right) & =\int_{\mathscr{M}} N\left(m^{\prime \prime}, m^{\prime}\right) M\left(m^{\prime}, m\right) d m^{\prime} \\
& =\int_{\mathscr{M}}\left|\left\langle m^{\prime \prime}, m^{\prime}\right\rangle\right|^{2}\left((n+1)^{2}\left|\left\langle m^{\prime}, m\right\rangle\right|^{2}-(n+2)\right) d m^{\prime} \\
& =(n+1)^{2} P_{\mathscr{M}}\left(m^{\prime \prime}, m, m^{\prime \prime}, m\right)-(n+2) \int_{\mathscr{M}}\left|\left\langle m^{\prime \prime}, m^{\prime}\right\rangle\right|^{2} d m^{\prime} \\
& =(n+1)\left(\left\langle m^{\prime \prime}, m^{\prime \prime}\right\rangle\langle m, m\rangle+\left\langle m^{\prime \prime}, m\right\rangle\left\langle m, m^{\prime \prime}\right\rangle\right)-(n+2) \\
& =(n+1)\left|\left\langle m^{\prime \prime}, m\right\rangle\right|^{2}-1 .
\end{aligned}
$$

Here we have used Corollary 1.15. Now for $v_{1}, v_{2} \in V$ let $w \in W$ be defined by

$$
w(m)=\left\langle v_{1}, m\right\rangle\left\langle m, v_{2}\right\rangle
$$

Then

$$
\begin{aligned}
\int_{\mathscr{M}} K\left(m^{\prime}, m\right) w\left(m^{\prime}\right) d m^{\prime} & =\int_{\mathscr{M}}\left((n+1)\left|\left\langle m^{\prime}, m\right\rangle\right|^{2}-1\right)\left\langle v_{1}, m^{\prime}\right\rangle\left\langle m^{\prime}, v_{2}\right\rangle d m^{\prime} \\
& =(n+1) P_{\mathscr{M}}\left(m, v_{1}, m, v_{2}\right)-\left\langle v_{1}, v_{2}\right\rangle \\
& =\left\langle v_{1}, m\right\rangle\left\langle m, v_{2}\right\rangle \\
& =w(m) .
\end{aligned}
$$


Now since elements of the form $\left\langle v_{1}, m\right\rangle\left\langle m, v_{2}\right\rangle$ span $W$, we see that $K\left(m^{\prime}, m\right)$ is the reproducing kernel with respect to $\langle,\rangle_{2}$ and so $\tilde{\zeta}=\tilde{\eta}^{-1}$.

COROLlaRY 3.7. Let $\mathscr{M}$ be a symmetric orbit with $|\mathscr{M}|=1$. Then the reproducing kernel of $W$ with respect to $\langle,\rangle_{2}$ is

$$
R_{2}\left(m^{\prime}, m\right)=(n+1)\left|\left\langle m^{\prime}, m\right\rangle\right|^{2}-1 .
$$

Now retuming to the general effective orbit $\mathscr{M}$, which we assume to be of modulus 1, we can transfer the natural algebra structure of End $V$ to $W$.

DEFINITION 3.8. Let $w_{1}, w_{2} \in W$ with $\sigma^{-1}\left(w_{i}\right)=T_{i} \in$ End $V, i=1,2$. Define $w_{1} \times w_{2} \in W$ by

$$
w_{1} \times w_{2}(m)=\sigma\left(T_{2} T_{1}\right)(m) \quad \text { for all } m \in \mathscr{M} .
$$

The algebra $(W, \times)$ thus defined is isomorphic to the full matrix algebra End $V$. Let $B_{1}\left(m, m_{1}, m_{2}\right)$ and $B_{2}\left(m, m_{1}, m_{2}\right)$ in $W^{(3)}$ be the triple kernels for $(W, \times)$ with respect to $\langle,\rangle_{1}$ and $\langle,\rangle_{2}$ respectively. We may utilise the results of Section 2 and obvious properties of the algebra End $V$ to deduce the following properties of $B_{1}$ and $B_{2}$.

PROPOSITION 3.9.

For all $m_{1}, m_{2}, m_{3}, m_{4} \in \mathscr{M}$, and $\alpha=1,2$,

(a) $\int_{\alpha} B_{\alpha}\left(m, m_{1}, m_{2}\right) B_{\alpha}\left(m_{4}, m, m_{3}\right) d m=\int_{\alpha} B_{\alpha}\left(m_{4}, m_{1}, m\right) B_{\alpha}\left(m, m_{2}, m_{3}\right) d m$

(b) $\int_{\alpha} B_{\alpha}\left(m, m_{1}, m_{2}\right) d m_{2}=R_{\alpha}\left(m_{1}, m\right)$ and

$$
\int_{\alpha} B_{\alpha}\left(m, m_{1}, m_{2}\right) d m_{1}=R_{\alpha}\left(m_{2}, m\right)
$$

(c) $B_{\alpha}\left(m, m_{2}, m_{1}\right)=\overline{B_{\alpha}\left(m, m_{1}, m_{2}\right)}$

(d) $\int_{1} B_{1}\left(m, m_{1}, m_{2}\right) d m=R_{1}\left(m_{2}, m_{1}\right)$

(e) $B_{1}\left(m, m_{1}, m_{2}\right)$ is symmetric.

This result suggests that in general $B_{1}$ will be a more natural object than $B_{2}$. Nevertheless we can compute $B_{2}$ in the case of $\mathscr{M}$ a symmetric orbit. 
PROPOSITION 3.10. Let $\mathscr{M}$ be a symmetric orbit with $|\mathscr{M}|=1$. Then for $m, m_{1}, m_{2} \in \mathscr{M}$,

$$
\begin{aligned}
B_{2}\left(m, m_{1}, m_{2}\right)=(n+1)^{2}\left\langle m, m_{1}\right\rangle\left\langle m_{1}, m_{2}\right\rangle\left\langle m_{2}, m\right\rangle & \\
& -(n+1)\left|\left\langle m, m_{1}\right\rangle\right|^{2}-(n+1)\left|\left\langle m, m_{2}\right\rangle\right|^{2}+1 .
\end{aligned}
$$

PROOF. Suppose $w_{1}, w_{2} \in W$ with $\sigma^{-1}\left(w_{i}\right)=T_{i} \in$ End $V, i=1,2$. Let $T_{3}=T_{2} T_{1}$ and let $K_{i}$ be the kernel of $T_{i}$ with respect to $\mathscr{M}$. By Proposition 1.16

$$
K_{i}\left(m_{1}, m_{2}\right)=\int_{\mathscr{M}}\left\langle T_{i} m, m\right\rangle\left((n+1)\left\langle m_{1}, m\right\rangle\left\langle m, m_{2}\right\rangle-\left\langle m_{1}, m_{2}\right\rangle\right) d m
$$

for $i=1,2$. Thus

$$
\begin{aligned}
K_{3}\left(m^{\prime \prime}, m\right)=\int_{\mathscr{M}} \int_{\mathscr{M}} \int_{\mathscr{M}}\left\langle T_{1} m_{1}, m_{1}\right\rangle\left((n+1)\left\langle m^{\prime \prime}, m_{1}\right\rangle\left\langle m_{1}, m^{\prime}\right\rangle-\left\langle m^{\prime \prime}, m^{\prime}\right\rangle\right) \\
\cdot\left\langle T_{2} m_{2}, m_{2}\right\rangle\left((n+1)\left\langle m^{\prime}, m_{2}\right\rangle\left\langle m_{2}, m\right\rangle-\left\langle m^{\prime}, m\right\rangle\right) d m_{1} d m_{2} d m^{\prime} \\
=\int_{\mathscr{M}} \int_{\mathscr{M}} w_{1}\left(m_{1}\right) w_{2}\left(m_{2}\right)\left((n+1)^{2}\left\langle m^{\prime \prime}, m_{1}\right\rangle\left\langle m_{1}, m_{2}\right\rangle\left\langle m_{2}, m\right\rangle\right. \\
-(n+1)\left\langle m^{\prime \prime}, m_{1}\right\rangle\left\langle m_{1}, m\right\rangle-(n+1)\left\langle m^{\prime \prime}, m_{2}\right\rangle\left\langle m_{2}, m\right\rangle \\
\left.+\left\langle m^{\prime \prime}, m\right\rangle\right) d m_{1} d m_{2} .
\end{aligned}
$$

Thus

$$
\begin{aligned}
w_{1} \times w_{2}(m) & =K_{3}(m, m) \\
& =\int_{\mathscr{M}} \int_{\mathscr{M}} w_{1}\left(m_{1}\right) w_{2}\left(m_{2}\right) B_{2}\left(m, m_{1}, m_{2}\right) d m_{1} d m_{2}
\end{aligned}
$$

where

$$
\begin{gathered}
B_{2}\left(m, m_{1}, m_{2}\right)=(n+1)^{2}\left\langle m, m_{1}\right\rangle\left\langle m_{1}, m_{2}\right\rangle\left\langle m_{2}, m\right\rangle-(n+1)\left|\left\langle m, m_{1}\right\rangle\right|^{2} \\
-(n+1)\left|\left\langle m, m_{2}\right\rangle\right|^{2}+1 .
\end{gathered}
$$

In contrast, the triple-kernel $B_{1}$ has a simple form even in the general case.

THEOREM 3.11. Let $\mathscr{M}$ be an effective orbit with $|\mathscr{M}|=1$. Then for $m, m_{1}, m_{2} \in \mathscr{M}$,

$$
B_{1}\left(m, m_{1}, m_{2}\right)=\left\langle m, m_{1}\right\rangle\left\langle m_{1}, m_{2}\right\rangle\left\langle m_{2}, m\right\rangle .
$$


ProOF. Let $T_{1}, T_{2} \in$ End $V$. Then for $v \in V$,

$$
\begin{aligned}
&\left\langle T_{2} T_{1} v, v\right\rangle=\int_{\mathscr{A}}\left\langle T_{1} v, m\right\rangle\left\langle T_{2} m, v\right\rangle d m \\
&=\int_{\mathscr{K}} \int_{\mathscr{M}}\left\langle\eta^{-1}\left(T_{1}\right) m_{1}, m_{1}\right\rangle\left\langle v, m_{1}\right\rangle\left\langle m_{1}, m\right\rangle d m_{1} \\
& \cdot \int_{\mathscr{A}}\left\langle\eta^{-1}\left(T_{2}\right) m_{2}, m_{2}\right\rangle\left\langle m, m_{2}\right\rangle\left\langle m_{2}, v\right\rangle d m_{2} d m \\
&=\iint_{\mathscr{M}}\left\langle\eta^{-1}\left(T_{1}\right) m_{1}, m_{1}\right\rangle\left\langle\eta^{-1}\left(T_{2}\right) m_{2}, m_{2}\right\rangle\left\langle v, m_{1}\right\rangle \\
& \cdot\left\langle m_{1}, m_{2}\right\rangle\left\langle m_{2}, v\right\rangle d m_{1} d m_{2} .
\end{aligned}
$$

Thus for $w_{1}, w_{2} \in W$,

$w_{1} \times w_{2}(m)=\int_{\mathscr{M}} \int_{\mathscr{M}} \tilde{\eta}^{-1}\left(w_{1}\right)\left(m_{1}\right) \tilde{\eta}^{-1}\left(w_{2}\right)\left(m_{2}\right)\left\langle m, m_{1}\right\rangle\left\langle m_{1}, m_{2}\right\rangle\left\langle m_{2}, m\right\rangle d m_{1} d m_{2}$.

But for $w, w^{\prime} \in W$,

$$
\left\langle\tilde{\eta}^{-1}(w), w^{\prime}\right\rangle_{2}=\left\langle w, w^{\prime}\right\rangle_{1}
$$

so

$$
w_{1} \times w_{2}(m)=\int_{1} \int_{1} w_{1}\left(m_{1}\right) w_{2}\left(m_{2}\right)\left\langle m, m_{1}\right\rangle\left\langle m_{1}, m_{2}\right\rangle\left\langle m_{2}, m\right\rangle d m_{1} d m_{2}
$$

for all $w_{1}, w_{2} \in W$. Thus

$$
B_{1}\left(m, m_{1}, m_{2}\right)=\left\langle m, m_{1}\right\rangle\left\langle m_{1}, m_{2}\right\rangle\left\langle m_{2}, m\right\rangle
$$

\section{Section 4}

Let $G$ be a compact Lie group and $(V, \rho)$ a finite-dimensional unitary representation of $G$ (not necessarily irreducible). Let $\Omega=\{v \in V \mid\langle v, v\rangle=1\}$ be the unit sphere, and suppose $\operatorname{dim} V=n$. Let $\mathfrak{g}$ be the Lie algebra of $G$ and $\mathfrak{g}^{*}$ its dual. We will continue to suppress the notation $\rho$, so that for $X \in \mathfrak{g}$ and $v \in V, \rho(X)(v)$ will be denoted simply by $X \cdot v$. 
DEFINITION 4.1. $\Phi: \Omega \rightarrow \mathfrak{g}^{*}$

$$
v \mapsto \Phi(v)(X)=\frac{1}{i}\langle X \cdot v, v\rangle
$$

for $v \in \Omega, X \in \mathfrak{g}$, is the moment map of $(V, \rho)$.

LEMMA 4.2. $\Phi$ is a $G$-map between $\Omega$ and $\mathfrak{g}^{*}$, where $G$ acts on $\mathfrak{g}^{*}$ via the co-adjoint action $\mathrm{Ad}^{*}$.

PROOF. For $g \in G$,

$$
\begin{aligned}
\Phi(g \cdot v)(X) & =\frac{1}{i}\langle X \cdot(g \cdot v), g \cdot v\rangle \\
& =\frac{1}{i}\left\langle g^{-1} \cdot(X \cdot(g \cdot v)), v\right\rangle \\
& =\frac{1}{i}\left\langle\operatorname{Ad}^{-1}(X) \cdot v, v\right\rangle \\
& =\Phi(v)\left(\operatorname{Ad} g^{-1}(X)\right) \\
& =\operatorname{Ad}^{*} g(\Phi(v))
\end{aligned}
$$

Let $\operatorname{Im} \Phi=\{\Phi(v) \mid v \in \Omega\}$ be the image of $\Phi$. This is a compact subset of $\mathfrak{g}^{*}$.

LEMMA 4.3. Let $\left(V_{1}, \rho_{1}\right)$ and $\left(V_{2}, \rho_{2}\right)$ be two unitary representations of $G$ with moment maps $\Phi_{1}$ and $\Phi_{2}$ respectively. Let $V=V_{1} \oplus V_{2}$ and $\Phi$ be the corresponding moment map. Then

$\operatorname{Im} \Phi=\left\{f \in \mathfrak{g}^{*} \mid f=t f_{1}+(1-t) f_{2}, \quad 0 \leq t \leq 1, f_{i} \in \operatorname{Im} \Phi_{i}, i=1,2\right\}$.

PROOF. If $\Omega_{1}, \Omega_{2}$ and $\Omega$ are the unit spheres of $V_{1}, V_{2}$ and $V$ respectively, then any $v \in \Omega$ can be written as $v=z_{1} v_{1}+z_{2} v_{2}$ with $v_{1} \in \Omega_{1}, v_{2} \in \Omega_{2}$ and $z_{1}, z_{2} \in \mathbb{C}$ satisfying $\left|z_{1}\right|^{2}+\left|z_{2}\right|^{2}=1$. Then for $X \in \mathfrak{g}$,

$$
\begin{aligned}
\Phi(v)(X) & =\frac{1}{i}\left\langle X \cdot\left(z_{1} v_{1}+z_{2} v_{2}\right), z_{1} v_{1}+z_{2} v_{2}\right\rangle \\
& =\left|z_{1}\right|^{2} \frac{1}{i}\left\langle X \cdot v_{1}, v_{1}\right\rangle+\left|z_{2}\right|^{2} \frac{1}{i}\left\langle X \cdot v_{2}, v_{2}\right\rangle
\end{aligned}
$$

since $\left\langle X \cdot v_{1}, v_{2}\right\rangle=\left\langle X \cdot v_{2}, v_{1}\right\rangle=0$. Thus

$$
\Phi(v)=\left|z_{1}\right|^{2} \Phi_{1}\left(v_{1}\right)+\left|z_{2}\right|^{2} \Phi_{2}\left(v_{2}\right) .
$$


LEMMA 4.4. Let $\left(V_{1}, \rho_{1}\right)$ and $\left(V_{2}, \rho_{2}\right)$ be two unitary representations of $G$ with moment maps $\Phi_{1}$ and $\Phi_{2}$ respectively. Let $V=V_{1} \otimes V_{2}$ and $\rho=\rho_{1} \otimes \rho_{2}$ the representation of $G \times G=G^{\prime}$ given by $\rho\left(g_{1}, g_{2}\right)\left(v_{1} \otimes v_{2}\right)=\left(g_{1} \cdot v_{1}\right) \otimes$ $\left(g_{2} \cdot v_{2}\right), g_{1}, g_{2} \in G$ and $v_{1}, v_{2} \in V$, with moment map $\Phi$. Then $\operatorname{Im} \Phi \subset \mathfrak{h}^{*} \times \mathfrak{g}^{*}$ satisfies

$$
\operatorname{Im} \Phi_{1} \times \operatorname{Im} \Phi_{2} \subset \operatorname{Im} \Phi \subset \operatorname{conv}\left(\operatorname{Im} \Phi_{1}\right) \times \operatorname{conv}\left(\operatorname{Im} \Phi_{2}\right)
$$

where conv $(S)$ means the convex hull of $S$.

PROOF. Let $\Omega_{1}, \Omega_{2}$ and $\Omega$ be the unit spheres of $V_{1}, V_{2}$ and $V$ respectively. Then for any $v_{1} \in \Omega_{1}, v_{2} \in \Omega_{2}, v=v_{1} \otimes v_{2} \in \Omega$ so that for any $\left(X_{1}, X_{2}\right) \in \mathfrak{g} \times \mathbf{g}$,

$$
\begin{aligned}
\Phi(v)\left(X_{1}, X_{2}\right) & =i^{-1}\left\langle\left(X_{1} \cdot v_{1}\right) \otimes v_{2}+v_{1} \otimes\left(X_{2} \cdot v_{2}\right), v_{1} \otimes v_{2}\right\rangle \\
& =i^{-1}\left(\left\langle X_{1} \cdot v_{1}, v_{1}\right\rangle\left\langle v_{2}, v_{2}\right\rangle+\left\langle v_{1}, v_{1}\right\rangle\left\langle X_{2} \cdot v_{2}, v_{2}\right\rangle\right) \\
& =\Phi_{1}\left(v_{1}\right)\left(X_{1}\right)+\Phi_{2}\left(v_{2}\right)\left(X_{2}\right) .
\end{aligned}
$$

Thus $\operatorname{Im} \Phi_{1} \times \operatorname{Im} \Phi_{2} \subset \operatorname{Im} \Phi$. Now let $\left\{v_{1}, \ldots, v_{n}\right\}$ be an orthonormal basis of $V_{1}$. Then if $v \in \Omega$, we may write $v=\sum_{k=1}^{n} v_{k} \otimes w_{k}$ where $w_{k} \in V_{2}$ with $\sum_{k=1}^{n}\left|w_{k}\right|^{2}=1$. Then

$$
\Phi(v)\left(X_{1}, X_{2}\right)=i^{-1} \sum_{k, l=1}^{n}\left\langle\left(X_{1} \cdot v_{k}\right) \otimes w_{k}+v_{k} \otimes\left(X_{2} \cdot w_{k}\right), v_{l} \otimes w_{l}\right\rangle
$$

so that

$$
\begin{aligned}
\Phi(v)\left(0, X_{2}\right) & =i^{-1} \sum_{k, l=1}^{n} \delta_{k, l}\left\langle\left(X_{2} \cdot w_{k}\right), w_{l}\right\rangle \\
& =i^{-1} \sum_{k=1}^{n}\left\langle\left(X_{2} \cdot w_{k}\right), w_{k}\right\rangle \\
& =i^{-1} \sum_{\substack{k=1 \\
\left|w_{k}\right| \neq 0}}^{n}\left|w_{k}\right|^{2}\left\langle X_{2} \cdot \frac{w_{k}}{\left|w_{k}\right|}, \frac{w_{k}}{\left|w_{k}\right|}\right\rangle \\
& =\left(\sum_{\substack{k=1 \\
\left|w_{k}\right| \neq 0}}^{n}\left|w_{k}\right|^{2} \Phi_{2}\left(u_{k}\right)\right)\left(X_{2}\right)
\end{aligned}
$$

where $u_{k}=w_{k} /\left|w_{k}\right|$ if $\left|w_{k}\right| \neq 0$ so that $u_{k} \in \Omega_{2}$.

Thus if $p_{2}: \mathfrak{g}^{*} \times \mathfrak{g}^{*} \rightarrow \mathfrak{g}^{*}$ is the projection onto the second factor, we see that $p_{2}(\operatorname{Im} \Phi) \subset \operatorname{conv}\left(\operatorname{Im} \Phi_{2}\right)$ so by symmetry $\operatorname{Im} \Phi \subset \operatorname{conv}\left(\operatorname{Im} \phi_{1}\right) \times$ conv $\left(\operatorname{Im} \Phi_{2}\right)$. 
LEMMA 4.5. Let $(V, \rho)$ be a unitary representation with moment map $\Phi$ and let $H$ be a Lie subgroup of $G$ with Lie algebra $\mathfrak{h}$. Let $p: \mathfrak{g}^{*} \rightarrow \mathfrak{h}^{*}$ be the projection dual to the inclusion $i: \mathfrak{h} \rightarrow \mathfrak{g}$. If $\Phi_{H}: \Omega \rightarrow \mathfrak{h}^{*}$ is the moment map of $\left(V,\left.\rho\right|_{H}\right)$ then $\Phi_{H}=p \circ \Phi$.

PROOF. Obvious from the definitions.

In general Im $\Phi$ need not be convex. For example, if $G=S U(2)$ and $(V, \rho)$, is the standard two-dimensional representation of $G$, then $\operatorname{Im} \Phi$ is easily seen to be a single $G$-orbit in $\mathfrak{g}^{*}$, namely a 2 -sphere. However we have the following.

LEMMA 4.6. Let $T$ be a torus with Lie algebra $\mathbf{t}$. Let $(V, \rho)$ be a finite dimensional representation of $T$ with weights $i \lambda_{1}, \ldots, i \lambda_{n} \in i t^{*}$. Then $\operatorname{Im} \Phi$ is the convex hull of $\left\{\lambda_{1}, \ldots, \lambda_{n}\right\} \subset \mathbf{t}^{*}$. In particular $\operatorname{Im} \Phi$ is convex.

PROOF. Write $V=V_{1} \oplus \ldots \oplus V_{n}$ with $V_{j}$ the one-dimensional representation with weight $i \lambda_{j}$. That is, for $X \in \mathfrak{t}$ and $v_{j} \in V_{j}, X \cdot v_{j}=i \lambda_{j}(X) v_{j}$. Now if $\Phi_{j}$ is the moment map of $V_{j}$ and if $v_{j} \in \Omega_{j}$, the unit sphere in $V_{j}$, we have

$$
\Phi_{j}\left(v_{j}\right)(X)=\frac{1}{i}\left\langle X \cdot v_{j}, v_{j}\right\rangle=\lambda_{j}(X) .
$$

Thus $\operatorname{Im} \Phi_{j}=\lambda_{j}$, and the result follows from Lemma 4.3 by induction.

Now let $T \subset G$ be a maximal torus with Lie algebra $t$. Choose a G-invariant positive-definite form (, ) on $\mathfrak{g}$. Let $i \lambda_{1}, \ldots, i \lambda_{n} \in i \mathfrak{t}^{*}$ be the weights of $(V, \rho)$ restricted to $T$ and let $D \subset \mathfrak{t}^{*}$ be the convex hull of $\left\{\lambda_{1}, \ldots, \lambda_{n}\right\}$. Then if $p: \mathfrak{g}^{*} \rightarrow \mathfrak{t}^{*}$ is the projection, Lemmas 4.5 and 4.6 imply immediately that

$$
p(\operatorname{Im} \Phi)=D .
$$

Denote by Ext $(D)$ those points of $D$ which are not contained in any open line segment in $D$. Clearly Ext $(D)$ is a subset of $\left\{\lambda_{1}, \ldots, \lambda_{n}\right\}$.

It will be useful to sometimes identify $\mathfrak{g}$ with $\mathfrak{g}^{*}$ via $($,$) and thus to consider$ $\mathfrak{t}^{*} \subset \mathbf{g}^{*}$.

LEMMA 4.7. If $f \in \operatorname{Im} \Phi \subset \mathfrak{g}^{*}$ and $p(f)=\lambda \in \operatorname{Ext}(D)$, then $f=\lambda$.

PROOF. Write $f=\lambda+\mu$ with $\mu \in \mathfrak{g}^{*}, \mu(\mathfrak{t})=0$. Let $\mathscr{O}$ be the co-adjoint orbit through $f$ and let $X \cdot f \in T \mathscr{O}_{f}$ with $X \in \mathfrak{g}$. Then $d p(X \cdot f)$ is a tangent 
vector to $\mathrm{t}^{*}$ at $p(f)=\lambda$. Now since $p(\mathscr{O}) \subset D$, any curve in $\mathscr{O}$ through $f$ with tangent vector $X \cdot f$ is sent by $p$ to a curve in $D$ through $\lambda \in \operatorname{Ext}(D)$. But any such curve must be singular since $D$ is a solid convex polyhedron in $\mathbf{t}^{*}$ with a finite number of vertices and $\lambda$ is such a vertex. Thus $p(X \cdot f)=0$, so that for all $Z \in \mathfrak{t}, X \in \mathfrak{g}, X \cdot f(Z)=0$.

Now a basic fact about Lie algebras of compact groups is that they are reductive. This implies that $\mathfrak{g}=\mathfrak{t} \oplus[\mathfrak{t}, \mathfrak{g}]$, an orthogonal direct sum with respect to the form $($,$) . Thus since we can rewrite the above as (\lambda+\mu)([Z, X])=0$, we get $\mu([Z, X])=0$ for all $Z \in \mathfrak{t}$ and $X \in \mathfrak{g}$ so that $\mu=0$ and $f=\lambda$.

LEMMA 4.8. Let $\lambda \in \operatorname{Ext}(D)$. Then $v \in \Omega$ is a weight vector for $T$ of weight $i \lambda$ if and only if $\Phi(v)=\lambda$.

PROOF. Suppose first that $v \in \Omega$ is a weight vector of weight $i \lambda$. Then $p(\Phi(v))=\lambda$ so that by the previous lemma, $\Phi(v)=\lambda$.

On the other hand let $v \in \Omega$ with $\Phi(v)=\lambda$. If $\left\{v_{1}, \ldots, v_{n}\right\}$ is an orthonormal basis of weight vectors with $v_{j}$ of weight $i \lambda_{j}$ then we can write $v=\sum_{i=1}^{n} \alpha_{i} v_{i}$ with $\sum_{i=1}^{n}\left|\alpha_{i}\right|^{2}=1$. Then if $Z \in \mathfrak{t}$,

$$
\begin{aligned}
\Phi(v)(Z) & =\frac{1}{i}\langle Z \cdot v, v\rangle \\
& =\frac{1}{i} \sum_{j=1}^{n}\left|\alpha_{j}\right|^{2}\left\langle Z \cdot v_{j}, v_{j}\right\rangle \\
& =\left(\sum_{j=1}^{n}\left|\alpha_{j}\right|^{2} \Phi\left(v_{j}\right)\right)(Z) \\
& =\left(\sum_{j=1}^{n}\left|\alpha_{j}\right|^{2} \lambda_{j}\right)(Z) .
\end{aligned}
$$

But if $\Phi(v)=\lambda$ then $\lambda=\sum_{j=1}^{n}\left|\alpha_{j}\right|^{2} \lambda_{j}$. Now since $\lambda \in \operatorname{Ext}(D)$ all the $\alpha_{j}$ must be zero except for one and so $v$ is actually a weight vector of weight $i \lambda$.

We remark more generally that if $F \subset D$ is a face of $D$ and

$$
F \cap\left\{\lambda_{1}, \ldots, \lambda_{n}\right\}=\left\{\lambda_{i_{1}}, \ldots, \lambda_{i_{k}}\right\}
$$

then the set of $v \in \Omega$ for which $p(\Phi(v)) \subset F$ is

$$
\left\{v=\left.\sum_{j=1}^{k} \alpha_{i_{j}} v_{i_{j}}\left|\sum_{j=1}^{k}\right| \alpha_{i_{j}}\right|^{2}=1\right\} .
$$


Now we assume henceforth that $G$ is semisimple and that $(V, \rho)$ is irreducible. Then it is well known that the weights $i \lambda_{1}, \ldots, i \lambda_{n}$ of $(V, \rho)$ are contained in the convex hull of the G-translates of one of them, say $i \lambda_{1}$. That is, if $\mathscr{O}$ is the co-adjoint orbit through $\lambda_{1}$, then $\mathscr{O}$ intersects $\mathfrak{t}$ in exactly Ext $(D)$, which is just the set of Weyl group translates of $\lambda_{1}$. From this it follows that $p(\mathscr{O}) \subset D$, which is part of a classical result of Kostant [12] which in this context is $p(\mathscr{O})=D$. The orbit $\mathscr{O}$ so determined will be called the extremal orbit of $\operatorname{Im} \Phi$, and since it is determined solely by the representation, it will be denoted $\mathscr{O}_{\rho}$.

The weight $i \lambda_{1}$ is usually called a highest weight and it is a standard fact that a weight vector $v_{1}$ for $i \lambda_{1}$ is unique up to a scalar.

PROPOSITION 4.9. Let $\Phi^{-1}\left(\mathscr{O}_{\rho}\right)=\mathscr{M}_{\rho} \subset \Omega$. Then $\mathscr{M}_{\rho} \rightarrow \mathscr{O}_{\rho}$ is an $S^{1}$ principal bundle.

PROOF. From the remark, $\Phi^{-1}\left(\lambda_{1}\right)=\left\{\left.z v_{1}|| z\right|^{2}=1\right\}$. But for $X \in \mathfrak{t}$,

$$
\exp t X \cdot v_{1}=e^{i t \lambda_{1}(X)} v_{1}
$$

so since $\Phi$ is a G-map by Lemma 4.2 , we have that $\mathscr{M}_{\rho}$ is a single G-orbit. Both $\mathscr{M}_{\rho}$ and $\mathscr{O}_{\rho}$ are homogeneous spaces for $G$ so $\Phi: \mathscr{M}_{\rho} \rightarrow \mathscr{O}_{\rho}$ is a fibre bundle with fibre $S^{1}$. But $S^{1}$, when regarded as the unit circle in $\mathbb{C}$, acts naturally on $V$ and preserves the fibre. We see that the $S^{1}$ action on a fibre arises from the group action of a one-parameter subgroup.

DEFINITION 4.10. The bundle $\Phi: \mathscr{M}_{\rho} \rightarrow \mathscr{O}_{\rho}$ will be called the canonical $S^{1}$ bundle of $(V, \rho)$.

THEOREM 4.11. The orbit $\mathscr{M}_{\rho} \subset \Omega$ is effective.

PROOF. We begin by recalling some basic facts about $G$ and the representation $(V, \rho)$.

Let $\mathfrak{g}$ be the complexification of $\mathfrak{g}$ and $\mathfrak{h} \subset \mathfrak{g}$ a Cartan subalgebra containing $\mathfrak{t}$. Let $\Delta$ be the set of roots of $\mathfrak{g}$ with respect to the action of $\mathfrak{h}$, and fix a subset $\Delta^{+} \subset \Delta$ of positive roots. For each $\alpha \in \Delta^{+}$, we may choose non-zero vectors $X_{\alpha}$ and $Y_{\alpha}$ in the root spaces for $\alpha$ and $-\alpha$ respectively such that $\left\langle X_{\alpha} \cdot v, w\right\rangle=\left\langle v, Y_{\alpha} \cdot w\right\rangle$ for all $v, w \in V$.

An element $\mu \in \mathfrak{h}^{*}$ is a weight of $\rho$ if for some non-zero vector $v \in V$ and all $H \in \mathfrak{h}, H \cdot v=\mu(H) v$. The set of all weights of $\rho$, denoted $\Lambda(\rho)$, is partially ordered by setting $\mu>\mu^{\prime}$ if $\mu=\mu^{\prime}+\sum_{i=1}^{k} m_{i} \alpha_{i}$, where $\alpha_{i} \in \Delta^{+}$ 
and the $m_{i} \geq 0$ are integers not all zero. Then $\Lambda(\rho)$ contains a unique highest weight $\mu_{1}$ with the property that $\mu_{1}>\mu$ for any other weight $\mu \in \Lambda(\rho)$. It is the extension to $h$ of the functional $i \lambda_{1}$ on $\mathrm{t}$, for some $\lambda_{1} \in \operatorname{Ext}(D)$.

For each $\mu \in \Lambda(\rho)$, we denote the weight space of $\mu$ by $V_{\mu}$ so that

$$
V=\oplus_{\mu \in \Lambda(\rho)} V_{\mu} .
$$

For $v_{1}, v_{2} \in V$, define $T_{v_{1}, v_{2}} \in$ End $V$ by

$$
T_{v_{1}, v_{2}}(v)=\left\langle v, v_{2}\right\rangle v_{1}
$$

For $\mu, \mu^{\prime} \in \Lambda(\rho)$ define $U_{\mu, \mu^{\prime}} \subset$ End $V$ to be the span of all $T_{v, v^{\prime}}$ with $v \in$ $V_{\mu}, v^{\prime} \in V_{\mu^{\prime}}$. Then clearly

$$
\text { End } V=\oplus_{\mu, \mu^{\prime} \in \Lambda(\rho)} U_{\mu, \mu^{\prime}} \text {. }
$$

The spaces $U_{\mu, \mu^{\prime}}$ can be partially ordered by setting $U_{\mu, \mu^{\prime}}>U_{\eta, \eta^{\prime}}$ if $\mu>\eta$ or if $\mu=\eta$ and $\mu^{\prime}>\eta^{\prime}$.

Let $\pi$ be the representation of $G$ on End $V$ by conjugation so that for $g \in$ $G, T \in$ End $V$ and $v \in V$,

$$
\pi(g)(T) v=g \cdot\left(T \cdot\left(g^{-1} \cdot v\right)\right)
$$

while if $X \in \mathfrak{g}$ then

$$
\pi(X)(T) v=X \cdot T \cdot v-T \cdot X \cdot v .
$$

For $\alpha \in \Delta^{+}, v \in V_{\mu}, v^{\prime} \in V_{\mu^{\prime}}, w \in V$,

$$
\begin{aligned}
\pi\left(X_{\alpha}\right) T_{v, v^{\prime}}(w) & =X_{\alpha} \cdot\left\langle w, v^{\prime}\right\rangle v-\left\langle X_{\alpha} \cdot w, v^{\prime}\right\rangle v \\
& =\left\langle w, v^{\prime}\right\rangle X_{\alpha} \cdot v-\left\langle w, Y_{\alpha} \cdot v^{\prime}\right\rangle v \\
& =T_{X_{\alpha} \cdot v, v^{\prime}}(w)-T_{v, Y_{\alpha} \cdot v^{\prime}}(w) .
\end{aligned}
$$

Thus

$$
\pi\left(X_{\alpha}\right) U_{\mu, \mu^{\prime}} \subset U_{\mu+\alpha, \mu^{\prime}} \oplus U_{\mu, \mu^{\prime}-\alpha} .
$$

Similarly

$$
\pi\left(Y_{\alpha}\right) T_{v, v^{\prime}}(w)=T_{Y_{\alpha} \cdot v, v^{\prime}}(w)-T_{v, X_{\alpha} \cdot v^{\prime}}(w)
$$

so that

$$
\pi\left(Y_{\alpha}\right) U_{\mu, \mu^{\prime}} \subset U_{\mu-\alpha, \mu^{\prime}} \oplus U_{\mu, \mu^{\prime}+\alpha}
$$


Now let

$$
U=\left\{T \in \text { End } V \mid\langle T m, m\rangle=0 \quad \forall m \in \mathscr{M}_{\rho}\right\} .
$$

We must show $U=0$. Clearly $U$ is invariant under $\pi(G)$ since $\mathscr{M}_{\rho}$ is a G-orbit, so it is also $\pi(\mathfrak{g})$ invariant. For any $T \in U$, we may write $T$ as

$$
T=\sum_{\mu, \mu^{\prime} \in \Lambda(\rho)} S_{\mu, \mu^{\prime}}
$$

where $S_{\mu, \mu^{\prime}} \in U_{\mu, \mu^{\prime}}$. Consider the set of all spaces $U_{\mu, \mu^{\prime}}$ for which there exists a $T \in U$ with non-zero component $S_{\mu, \mu^{\prime}}$ in $U_{\mu, \mu^{\prime}}$.

We will show this set to be empty. If not, let $U_{\eta, \eta^{\prime}}$ be a maximal element, so that there exists $T \in U$ with

$$
T=S_{\eta, \eta^{\prime}}+\sum_{\substack{\mu, \mu^{\prime} \in \Lambda(\rho) \\\left(\mu, \mu^{\prime}\right) \neq\left(\eta, \eta^{\prime}\right)}} S_{\mu, \mu^{\prime}}
$$

where $0 \neq S_{\eta, \eta^{\prime}} \in U_{\eta, \eta^{\prime}}$ and $S_{\mu, \mu^{\prime}} \in U_{\mu, \mu^{\prime}}$ for all $\mu, \mu^{\prime} \in \Lambda(\rho)$.

We claim that $\eta=\eta^{\prime}=\mu_{1}$. For suppose first that $\mu_{1}>\eta$. Write

$$
S_{\eta, \eta^{\prime}}=\sum_{l=1}^{s} T_{w_{l}, w_{l}^{\prime}}
$$

where $\left\{w_{1}^{\prime}, \ldots, w_{s}^{\prime}\right\}$ is a basis of $V_{\eta^{\prime}}$ and $w_{\ell} \in V_{\eta}$, with say $w_{1} \neq 0$. Then since $(V, \rho)$ is irreducible, we can find $\alpha \in \Delta^{+}$such that $X_{\alpha} \cdot w_{1} \neq 0$. Thus

$$
\pi\left(X_{\alpha}\right) S_{\eta, \eta^{\prime}}=\sum_{l=1}^{s}\left(T_{X_{\alpha} \cdot w_{l}, w_{l}^{\prime}}-T_{w_{l}, Y_{\alpha} \cdot w_{l}^{\prime}}\right)
$$

has a nonzero component in $U_{\eta+\alpha, \eta^{\prime}}$ since $\left\{w_{1}^{\prime}, \ldots, w_{s}^{\prime}\right\}$ is linearly independent.

Combining this with the fact that none of the elements $\pi\left(X_{\alpha}\right) S_{\mu, \mu^{\prime}},\left(\mu, \mu^{\prime}\right) \neq$ $\left(\eta, \eta^{\prime}\right)$, can have a component in $U_{\eta+\alpha, \eta^{\prime}}$ (by the maximality of $U_{\eta, \eta^{\prime}}$ and (4.1)), we find that $\pi\left(X_{\alpha}\right) T$ has a non-zero component in $U_{\eta+\alpha, \eta^{\prime}}$. But this is impossible since $\pi\left(X_{\alpha}\right) T \in U$, so we must have $\eta=\mu_{1}$.

Now suppose that $\mu_{1}>\eta^{\prime}$. Then we write

$$
S_{\eta, \eta^{\prime}}=T_{v_{1}, v^{\prime}}
$$

where $v_{1} \in V_{\eta}=V_{\mu_{1}}$ and $v^{\prime} \in V_{\eta^{\prime}}$, with both $v_{1}, v^{\prime}$ non-zero. This is possible since $V_{\mu_{1}}$ is one-dimensional. Now again by the irreducibility of $(V, \rho)$, we may find $\beta \in \Delta^{+}$such that $X_{\beta} \cdot v^{\prime} \neq 0$. Then

$$
\pi\left(Y_{\beta}\right) S_{\eta, \eta^{\prime}}=T_{Y_{\beta} \cdot v_{1}, v^{\prime}}-T_{v_{1}, X_{\beta} \cdot v^{\prime}}
$$


so that $\pi\left(Y_{\beta}\right) T$ has a non-zero component in $U_{\eta, \eta^{\prime}+\alpha}$. But since $\pi\left(Y_{\beta}\right) T \in U$, we have a contradiction so that $\eta^{\prime}=\mu_{1}$.

We have thus shown that if $U \neq 0$, then there exists $T \in U$ with

$$
T=S_{\mu_{1}, \mu_{1}}+\sum_{\substack{\mu, \mu^{\prime} \in \Lambda(\rho) \\\left(\mu, \mu^{\prime}\right) \neq\left(\mu_{1}, \mu_{1}\right)}} S_{\mu, \mu^{\prime}}
$$

where $0 \neq S_{\mu_{1}, \mu_{1}} \in U_{\mu_{1}, \mu_{1}}$ and $S_{\mu, \mu^{\prime}} \in U_{\mu, \mu^{\prime}}$ for $\left(\mu, \mu^{\prime}\right) \neq\left(\mu_{1}, \mu_{1}\right)$. Now let $v_{1} \in \Omega$ be a weight vector for $\mu_{1}$, that is, $v_{1} \in V_{\mu_{1}}$. Then $\Phi\left(v_{1}\right)=\lambda_{1} \in \mathscr{O}_{\rho}$ so that $v_{1} \in M_{\rho}$. But then

$$
\begin{aligned}
\left\langle T v_{1}, v_{1}\right\rangle & =\left\langle S_{\mu_{1}, \mu_{1}} v_{1}, v_{1}\right\rangle+\sum_{\substack{\mu, \mu^{\prime} \in \Lambda(\rho) \\
\left(\mu, \mu^{\prime}\right) \neq\left(\mu_{1}, \mu_{1}\right)}}\left\langle S_{\mu, \mu^{\prime}} v_{1}, v_{1}\right\rangle \\
& =\left\langle S_{\mu_{1}, \mu_{1}} v_{1}, v_{1}\right\rangle \\
& \neq 0 .
\end{aligned}
$$

This is a contradiction, so that $U=0$ and $\mathscr{M}_{\rho}$ is effective.

\section{Section 5}

Recall that the co-adjoint orbit $\mathscr{O}_{\rho}$ is canonically a symplectic manifold, that is, it carries a closed non-degenerate 2-form $\omega$. For $f \in \mathscr{O}_{\rho}$, let $X_{1} \cdot f, X_{2} \cdot f \in$ $T \mathscr{O}_{\rho}(f)$ where $X_{1}, X_{2} \in \mathfrak{g}$. Then

$$
\omega_{f}\left(X_{1} \cdot f, X_{2} \cdot f\right)=f\left(\left[X_{1}, X_{2}\right]\right) .
$$

On the other hand, the complex structure of $V$ defines the 1-form $\theta$ on $V$ by

$$
\theta_{v}(w)=\operatorname{Im}\langle w, v\rangle
$$

for $v \in V, w \in T V(v) \simeq V$. Then for $v \in V, w_{1}, w_{2} \in T V(v)$,

$$
d \theta_{v}\left(w_{1}, w_{2}\right)=2 \operatorname{Im}\left\langle w_{1}, w_{2}\right\rangle
$$

This is the 2-form on $V$ which determines its symplectic structure. Both $\theta$ and $d \theta$ may be restricted to $\mathscr{M}_{\rho} \subset V$.

LEMMA 5.1. On $\mathscr{M}_{\rho}, d \theta=(d \Phi)^{*}(\omega)$. 
PROOF. Let $m \in \mathscr{M}_{\rho}$ and $X_{1} \cdot m, X_{2} \cdot m \in T \mathscr{M}_{\rho}(m)$, where $X_{1}, X_{2} \in \mathfrak{g}$. Then

$$
\begin{aligned}
d \theta_{m}\left(X_{1} \cdot m, X_{2} \cdot m\right) & =2 \operatorname{Im}\left\langle X_{1} \cdot m, X_{2} \cdot m\right\rangle \\
& =\frac{1}{i}\left(\left\langle X_{1} \cdot m, X_{2} \cdot m\right\rangle-\left\langle X_{2} \cdot m, X_{1} \cdot m\right\rangle\right) \\
& =\frac{1}{i}\left\langle\left[X_{1}, X_{2}\right] \cdot m, m\right\rangle \\
& =\Phi(m)\left(\left[X_{1}, X_{2}\right]\right) \\
& =\omega_{f}\left(X_{1} \cdot f, X_{2} \cdot f\right)
\end{aligned}
$$

where $f=\Phi(m) \in \mathscr{O}_{\rho}$. But $\Phi$ is a G-map, so that $d \Phi\left(X_{i} \cdot m\right)=X_{i} \cdot f$, $i=1,2$ so that $d \theta=(d \Phi)^{*}(\omega)$.

Note that the 1 -form $\theta$, when restricted to $\mathscr{M}_{\rho}$, is a connection 1 -form for the canonical bundle $\Phi: \mathscr{M}_{\rho} \rightarrow \mathscr{O}_{\rho}$ whose curvature 2-form, by the Lemma above, is $\omega$. Thus the familiar connection and curvature on the quantum bundle in geometric quantization appear here naturally as manifestations of the complex structure of $V$.

The orbit $\mathscr{M}_{\rho}$ carries a space $W$ of symbols where as before

$$
W=\left\{\sigma_{T} \mid T \in \text { End } V\right\}
$$

and by Theorem 4.11, $\sigma:$ End $V \rightarrow W$ is an isomorphism. As in Section 3, we have two inner products $\langle,\rangle_{1}$ and $\langle,\rangle_{2}$ on $W$, as well as the algebra structure $(W, x)$.

We now make a simple but crucial observation. Each function $\sigma_{T} \in W$ has the property that $\sigma_{T}(z m)=\sigma_{T}(m)$ for all $z \in S^{1}$, so that $W$ really 'lives' on the orbit $\mathscr{O}_{\rho}$.

DEFINITION 5.2. For $T \in$ End $V$, let $a_{T}$ be the function on $\mathscr{O}_{\rho}$ defined by

$$
a_{T}(f)=\sigma_{T}\left(\Phi^{-1}(f)\right)
$$

for $f \in \mathscr{O}$. This function will be called the symbol of $T$. Let $A_{\rho}$ be the space of all $a_{T}, T \in$ End $V$, and $a:$ End $V \rightarrow A_{\rho}$ the map that sends $T$ to $a_{T}$.

LEMMA 5.3. $a:$ End $V \rightarrow A_{\rho}$ is an isomorphism. 
Now let $d \mu$ be the unique G-invariant measure on $\theta_{\rho}$ such that

$$
\int_{\sigma_{\rho}} \phi(f) d \mu(f)=\int_{\mathscr{A}_{\rho}} \phi(\Phi(m)) d m
$$

for all continuous functions $\phi$ on $\mathscr{O}_{\rho}$. Note that $d \mu$ may also be specified by requiring that it be G-invariant and that $\int_{\sigma_{\rho}} d \mu=n$ (this follows from Corollary 1.7).

Define inner products $\langle,\rangle_{\alpha}, \alpha=1,2$ on $A_{\rho}$ by

$$
\left\langle a_{T_{1}}, a_{T_{2}}\right\rangle_{\alpha}=\left\langle\sigma_{T_{1}}, \sigma_{T_{2}}\right\rangle_{\alpha}
$$

for all $T_{1}, T_{2} \in$ End $V$. Let $\eta^{\prime}: A_{\rho} \rightarrow A_{\rho}$ be the unique invertible operator such that for all $a_{1}, a_{2} \in A_{\rho}$,

$$
\left\langle\eta^{\prime}\left(a_{1}\right), a_{2}\right\rangle_{1}=\left\langle a_{1}, a_{2}\right\rangle_{2} .
$$

Equivalently we may say that for all $T \in$ End $V, a_{\eta(T)}=\eta^{\prime}\left(a_{T}\right)$.

We let $N^{\prime} \in A_{\rho}^{(2)}$ be the kemel of $\eta^{\prime}$ with respect to $(,)_{2}$, so that for all $a \in A_{\rho}$ and $f \in \mathscr{O}_{\rho}$,

$$
\eta^{\prime}(a)(f)=\int_{\theta_{p}} N^{\prime}\left(f^{\prime}, f\right) a\left(f^{\prime}\right) d \mu\left(f^{\prime}\right)
$$

The kernel of $\left(\eta^{\prime}\right)^{-1}: A_{\rho} \rightarrow A_{\rho}$ with respect to $\langle,\rangle_{2}$ will be denoted $M^{\prime} \in A_{\rho}^{(2)}$. The reproducing kernel for $A_{\rho}$ with respect to $\langle,\rangle_{\alpha}$ will be denoted $R_{\alpha}^{\prime}, \alpha=1,2$.

DEFINITION 5.4. For $a_{T_{1}}, a_{T_{2}} \in A_{\rho}\left(T_{1}, T_{2} \in\right.$ End $\left.V\right)$ we define the *-product of $a_{T_{1}}$ and $a_{T_{2}}$ to be

$$
a_{T_{1}} * a_{T_{2}}=a_{T_{2} T_{1}} .
$$

The triple-kernel for the algebra $\left(A_{\rho}, *\right)$ with respect to $\langle,\rangle_{\alpha}$ will be denoted $B_{\alpha}\left(f, f_{1}, f_{2}\right), \alpha=1,2$. The following is a direct consequence of Propositions $2.7,2.9,2.10,2.11$ and 2.12 and elementary properties of End $V$.

\section{PROPOSITION 5.5.}

For all $f_{1}, f_{2}, f_{3}, f_{4} \in \mathscr{O}_{\rho}, \alpha=1,2$,

(a) $\int_{\alpha} B_{\alpha}^{\prime}\left(f, f_{1}, f_{2}\right) B_{\alpha}^{\prime}\left(f_{4}, f, f_{3}\right) d \mu(f)=\int_{\alpha} B_{\alpha}^{\prime}\left(f_{4}, f_{1}, f\right) B_{\alpha}^{\prime}\left(f, f_{2}, f_{3}\right) d \mu(f)$ 
(b) $\int_{\alpha} B_{\alpha}^{\prime}\left(f, f_{1}, f_{2}\right) d \mu\left(f_{2}\right)=R_{\alpha}^{\prime}\left(f_{1}, f\right)$ and $\int_{\alpha} B_{\alpha}^{\prime}\left(f, f_{1}, f_{2}\right) d \mu\left(f_{1}\right)=R_{\alpha}^{\prime}\left(f_{2}, f\right)$

(c) $B_{\alpha}^{\prime}\left(f, f_{2}, f_{1}\right)=\overline{B_{\alpha}^{\prime}\left(f, f_{1}, f_{2}\right)}$

(d) $\int_{1} B_{1}^{\prime}\left(f, f_{1}, f_{2}\right) d \mu(f)=R_{1}^{\prime}\left(f_{1}, f_{2}\right)$

(e) $B_{1}^{\prime}\left(f, f_{1}, f_{2}\right)$ is symmetric.

\section{Section 6}

We now construct the Fourier transform for $G$, a compact semisimple Lie group. For each $\rho \in \hat{G}$, the unitary dual of $G$, we have the canonical $S^{1}$ bundle $\Phi: \mathscr{M}_{\rho} \rightarrow \mathscr{O}_{\rho}$, with $\mathscr{O}_{\rho}$ the extremal orbit in $\mathfrak{g}^{*}$. Since $\hat{G}$ is parametrized by the set of highest weights, it follows from the discussion in Section 4 that a representation $\rho \in \hat{G}$ is uniquely determined by the extremal orbit $\mathscr{O}_{\rho}$. Define $\mathfrak{g}_{\mathrm{INT}}^{*} \subset \mathfrak{g}^{*}$ to be the union of all extremal orbits

$$
\mathfrak{g}_{\mathrm{INT}}^{*}=\cup_{\rho \in \hat{G}} \mathscr{O}_{\rho}
$$

We use the subscript INT since the set of extremal orbits is the same as the set of integral orbits, in the sense of geometric quantization (see Kostant [11]).

The cotangent bundle $T^{*} G$ of $G$ can be identified with $G \times \mathfrak{g}^{*}$ by right translation. Define $T^{*} G_{\mathrm{INT}}$, the integral cotangent bundle, to be

$$
T^{*} G_{\mathrm{INT}}=G \times g_{\mathrm{INT}}^{*} \subset T^{*} G .
$$

DEFINITION 6.1. The Fourier kernel of $G$ is the function $e$ on $T^{*} G_{\mathrm{INT}}$ defined by

$$
e(g, f)=\left\langle g \cdot \Phi^{-1}(f), \Phi^{-1}(f)\right\rangle
$$

where $g \in G, f \in \mathscr{O}_{\rho} \subset \mathfrak{g}_{\mathrm{INT}}^{*}$, and $\Phi: \mathscr{M}_{\rho} \rightarrow \mathscr{O}_{\rho}$ is the canonical $S^{1}$ bundle of $\rho$.

It is clear that $e$ is well-defined and is a continuous function on $T^{*} G_{\mathrm{INT}}$. Also note that

$$
e(g, f)=e\left(g, g^{\prime} \cdot f\right)
$$

whenever $g$ and $g^{\prime}$ in $G$ commute. Thus $e$ does not depend on our choice of identification of $T^{*} G$ with $G \times \mathfrak{g}^{*}$. 
DEFINITION 6.2. The Fourier transform of a function $\phi \in L^{1}(G)$ is the function $F \phi$ on $\mathfrak{g}_{\mathrm{INT}}^{*}$ defined by

$$
F \phi(f)=\int_{G} \phi(g) e(g, f) d g
$$

where $f \in \mathfrak{g}_{\mathrm{INT}}^{*}$.

Here we have chosen Haar measure $d g$ on $G$ so that $\int_{G} d g=1$.

Now fix an irreducible unitary representation $(V, \rho)$ with extremal orbit $\mathscr{O}_{\rho}$ and $a:$ End $V \rightarrow A_{\rho}$ as in Section 5.

LEMMA 6.3. If $\phi \in L^{1}(G)$, then $\left.F \phi\right|_{\mathscr{O}_{\rho}}=a_{\rho(\phi)}$ where

$$
\rho(\phi)=\int_{G} \phi(g) \rho(g) d g \in \text { End } V .
$$

PROOF. Follows immediately from the definitions.

Now since any $T \in$ End $V$ can be written as $\rho(\phi)$ for some $\phi \in L^{1}(G)$, we see that the image of $F$ restricted to $\mathscr{O}_{\rho} \subset \mathfrak{g}_{\mathrm{INT}}^{*}$ is exactly $A_{\rho}$.

For $v_{1}, v_{2} \in V$, define the matrix coefficient $u_{v_{1}, v_{2}} \in L^{1}(G)$ by

$$
u_{v_{1}, v_{2}}(g)=\left\langle v_{1}, g \cdot v_{2}\right\rangle
$$

for $g \in G$, and let $U_{\rho}$ be the space of all such functions.

LEMMA 6.4. For $v_{1}, v_{2} \in V$, let $T_{v_{1}, v_{2}} \in$ End $V$ be defined by $T_{v_{1}, v_{2}}(v)=$ $\left\langle v, v_{2}\right\rangle v_{1}$. Then

$$
\left.F u_{v_{1}, v_{2}}\right|_{\theta_{\rho}}=\frac{1}{n} a_{T_{v_{1}, v_{2}}} .
$$

PROOF. Let $f \in \mathscr{O}_{\rho}$ and $m \in \mathscr{M}_{\rho}$ such that $\Phi(m)=f$. Then

$$
\begin{aligned}
F u_{v_{1}, v_{2}}(f) & =\int_{G} u_{v_{1}, v_{2}}(g)\langle g \cdot m, m\rangle d g \\
& =\int_{G} \overline{\left\langle g \cdot v_{2}, v_{1}\right\rangle}\langle g \cdot m, m\rangle d g \\
& =\frac{1}{n}\left\langle v_{1}, m\right\rangle\left\langle m, v_{2}\right\rangle \\
& =\frac{1}{n}\left\langle T_{v_{1}, v_{2}}(m), m\right\rangle \\
& =\frac{1}{n} a_{T_{v_{1}, v_{2}}}(f)
\end{aligned}
$$


where we have used the Schur orthogonality relations.

LEMMA 6.5. Let $\phi \in L^{1}(G)$ such that $\phi \in U_{\rho}^{\perp}$. Then $\left.F \phi\right|_{\rho_{\rho}}=0$.

PROOF. If $\phi \in U_{\rho}^{\perp}$ then for $f \in \mathscr{O}_{\rho}$ and $m \in \mathscr{M}_{\rho}$ with $\Phi(m)=f$, we have

$$
\begin{aligned}
F \phi(f) & =\int_{G} \phi(g)\langle g \cdot m, m\rangle d g \\
& =\int_{G} \phi(g) \overline{u_{m, m}(g)} d g \\
& =0
\end{aligned}
$$

Now let $\chi_{\rho}$ be the character of $\rho$, so that for $g \in G$,

$$
\chi_{\rho}(g)=\operatorname{tr} \rho(g) .
$$

The following is our version of the Kirillov character formula in this setting.

THEOREM 6.6. $\bar{\chi}_{\rho} \in U_{\rho}$ and $F \bar{\chi}_{\rho}(f)=1 / n$ for all $f \in \mathscr{O}_{\rho}$.

PROOF. If $\left\{v_{1}, \ldots, v_{n}\right\}$ is an orthonormal basis of $V$ then for $g \in G$,

$$
\begin{aligned}
\chi_{\rho}(g) & =\sum_{i=1}^{n}\left\langle g \cdot v_{i}, v_{i}\right\rangle \\
& =\sum_{i=1}^{n} \overline{u_{v_{i}, v_{i}}(g)}
\end{aligned}
$$

Thus $\bar{\chi}_{\rho} \in U_{\rho}$. Now

$$
\begin{aligned}
F \bar{\chi}_{\rho} & =\sum_{i=1}^{n} F u_{v_{i}, v_{i}} \\
& =\frac{1}{n} \sum_{i=1}^{n} a_{T_{v_{i}, v_{i}}} \\
& =\frac{1}{n} a_{I} \\
& =\frac{1}{n}
\end{aligned}
$$

where we have used the notation and result of Lemma 6.4 and where $I \in$ End $V$ is the identity. 
THEOREM 6.7. For any $\phi \in L^{1}(G)$,

$$
\operatorname{tr} \rho(\phi)=\int_{\sigma_{\rho}} F \phi(f) d \mu(f) .
$$

ProOF.

$$
\begin{aligned}
\operatorname{tr} \rho(\phi) & =\int_{\mathscr{A}_{\rho}}\langle\rho(\phi) m, m\rangle d m \\
& =\int_{\theta_{\rho}} a_{\rho(\phi)}(f) d \mu(f) \\
& =\int_{\theta_{\rho}} F \phi(f) d \mu(f)
\end{aligned}
$$

where we have used Proposition 1.5.

PROPOSITION 6.8. Let $u \in U_{\rho}$ be a positive-definite function. Then $F u(f)$ $\geq 0$ for all $f \in \mathscr{O}_{\rho}$.

PROOF. It is a standard fact that any $u_{v, v} \in U_{\rho}$ is positive-definite and any $u \in U_{\rho}$ which is positive-definite can be written as

$$
u=\sum_{i=1}^{r} u_{w_{i}, w_{i}}
$$

with $w_{i} \in V, i=1, \ldots, r$. Then for $f \in \mathscr{O}_{\rho}$ and $m \in \mathscr{M}_{\rho}$ with $\Phi(m)=f$, we have

$$
\begin{aligned}
F u(f) & =\sum_{i=1}^{r} F u_{w_{i}, w_{i}}(f) \\
& =\frac{1}{n} \sum_{i=1}^{r} a_{T_{w_{i}, w_{i}}}(f) \\
& =\frac{1}{n} \sum_{i=1}^{r}\left|\left\langle w_{i}, m\right\rangle\right|^{2} \geq 0
\end{aligned}
$$

from Lemma 6.4. 
Now for $\phi_{1}, \phi_{2} \in L^{1}(G)$, define their convolution

$$
\phi_{1} * \phi_{2}(g)=\int_{G} \phi_{1}\left(g g_{0}^{-1}\right) \phi_{2}\left(g_{0}\right) d g_{0}
$$

Then $\rho\left(\phi_{1} * \phi_{2}\right)=\rho\left(\phi_{2}\right) \rho\left(\phi_{1}\right)$ and from Lemma 6.3 and Definition 5.4 we get

PROPOSITION 6.9. For $\phi_{1}, \phi_{2} \in L^{1}(G)$,

$$
\left.F\left(\phi_{1} * \phi_{2}\right)\right|_{\sigma_{\rho}}=\left.\left.F\left(\phi_{1}\right)\right|_{\sigma_{\rho}} * F\left(\phi_{2}\right)\right|_{\sigma_{\rho}} .
$$

Thus the *-product on $A_{\rho}$ is the 'Fourier transform side' of convolution in $U_{\rho}$.

\section{Section 7}

We now examine in some detail the case of $G=S U(2)$. Notation and the basic facts about the representation theory are taken from Vilenkin [20]. We first review these facts. The representations in $\hat{G}$ are indexed by $\ell=0,1 / 2,1, \ldots$ and denoted $\rho_{\ell}$. Let $V_{\ell}$ be the space of polynomials in one variable of degree $2 \ell$. Then if $\phi \in V_{\ell}$ and

$$
g=\left|\begin{array}{cc}
\alpha & \beta \\
-\bar{\beta} & \bar{\alpha}
\end{array}\right|
$$

then $\rho_{\ell}(g) \phi(x)=(\beta x+\bar{\alpha})^{2 \ell} \phi((\alpha x-\bar{\beta}) /(\beta x+\bar{\alpha}))$. Let

$$
X_{1}=\frac{1}{2}\left|\begin{array}{cc}
0 & i \\
i & 0
\end{array}\right|, \quad X_{2}=\frac{1}{2}\left|\begin{array}{cc}
0 & -1 \\
1 & 0
\end{array}\right|, \quad X_{3}=\frac{1}{2}\left|\begin{array}{cc}
i & 0 \\
0 & -i
\end{array}\right| .
$$

Then $\left\{X_{1}, X_{2}, X_{3}\right\}$ is a basis of $\mathfrak{g}$ and $\left[X_{1}, X_{2}\right]=X_{3},\left[X_{2}, X_{3}\right]=X_{1}$ and $\left[X_{3}, X_{1}\right]=X_{2}$. A basis for $V_{\ell}$ consists of the monomials

$$
\Psi_{n}(x)=\frac{x^{\ell-n}}{\sqrt{(\ell-n) !(\ell+n) !}} \quad \text { for } \quad-\ell \leq n \leq \ell .
$$

This basis is orthonormal and the action of the Lie algebra is given by the formulae

$$
\begin{aligned}
& \rho\left(X_{1}\right) x^{\ell-n}=\frac{i}{2}(\ell-n) x^{\ell-n-1}+\frac{i}{2}(\ell+n) x^{\ell-n+1} \\
& \rho\left(X_{2}\right) x^{\ell-n}=\frac{1}{2}(\ell-n) x^{\ell-n-1}-\frac{1}{2}(\ell+n) x^{\ell-n+1} \\
& \rho\left(X_{3}\right) x^{\ell-n}=-i n x^{\ell-n} .
\end{aligned}
$$


We denote the unit sphere in $V_{\ell}$ by $\Omega_{\ell}$ and $\Phi_{\ell}: \Omega_{\ell} \rightarrow \mathfrak{g}^{*}$ the moment map for $\rho_{\ell}$. From the above equations we have

LEMMA 7.1.

$$
\Phi_{\ell}\left(\Psi_{n}\right)=-n X_{3}^{*} \text { for } \quad-\ell \leq n \leq \ell
$$

Let $f_{\ell}=\ell X_{3}^{*} \in \mathfrak{g}^{*}$, and $\mathscr{O}_{\ell}$ the orbit through $f_{\ell}$. Then $\mathscr{O}_{\ell}$ is the extremal orbit for the representation $\rho_{\ell}$ and is simply a 2-sphere. Then for $g \in G$,

$$
e\left(g, f_{\ell}\right)=\left\langle g \cdot \Psi_{-\ell}, \Psi_{-\ell}\right\rangle \text {. }
$$

LEMMA 7.2. For

$$
g=\left|\begin{array}{rr}
\alpha & \beta \\
-\bar{\beta} & \bar{\alpha}
\end{array}\right| \in G, \quad e\left(g, f_{\ell}\right)=\alpha^{2 \ell} .
$$

PROOF. From Vilenkin [20], we have

$$
\begin{aligned}
e\left(g, f_{\ell}\right) & =t_{-\ell-\ell}^{\ell}(g)=\frac{1}{(2 \ell) !} \frac{1}{\alpha^{-2 \ell}} \frac{d^{2 \ell}}{d z^{2 \ell}}\left(z^{2 \ell}\right) \\
& =\alpha^{2 \ell}
\end{aligned}
$$

Introduce the positive definite form on $\mathfrak{g}$

$$
(X, Y)=-2 \operatorname{tr}(X Y)
$$

for $X, Y \in \mathfrak{g}=S U(2)$. The basis $\left\{X_{1}, X_{2}, X_{3}\right\}$ is thus orthonormal. The orbit $\mathscr{O}_{\ell}$ is the sphere of radius $\ell$ when $\mathfrak{g}$ is identified with $\mathfrak{g}^{*}$ by the above form.

DEFINITION 7.3. For

$$
g=\left|\begin{array}{rr}
\alpha & \beta \\
-\bar{\beta} & \bar{\alpha}
\end{array}\right| \in G
$$

define $C(g) \in \mathfrak{g}$ by

$$
C(g)=\left|\begin{array}{rr}
\frac{\bar{\alpha}-\alpha}{2} & -\beta \\
\bar{\beta} & \frac{\alpha-\bar{\alpha}}{2}
\end{array}\right| .
$$


LEMMA 7.4. For

$$
g=\left|\begin{array}{rr}
\alpha & \beta \\
-\bar{\beta} & \bar{\alpha}
\end{array}\right| \in G,
$$

let $d=\operatorname{det}(I+g) \neq 0$. Here $I$ is the identity matrix. Then

$$
C(g)=\frac{d}{2} \frac{I-g}{I+g} .
$$

We note that $d=\operatorname{det}(I+g)=2+\operatorname{tr} g$. The map $g \rightarrow(I-g) /(I+g)$ is the well-known Cayley transform. Because of the possible singularity when $\operatorname{tr} g=-2$, it is defined only on a subset of $G$. Note however that the map $C: G \rightarrow \mathfrak{g}$ has no such problem and is defined on all of $G$.

We also remark that the Cayley transform has been shown to play an important role in the study of the Fourier transform for certain nilpotent Lie groups (see Howe, Ratcliff and Wildberger [7] and Wildberger [22]).

THEOREM 7.5. For $g \in G$ and $X \in \mathscr{O}_{\ell} \subset \mathfrak{g}$,

$$
e(g, X)=\left(\frac{\operatorname{tr} g}{2}+\frac{i}{2 \ell}(C(g), X)\right)^{2 \ell} .
$$

PRoOF. Since $X \in \mathscr{O}_{\ell}, \quad X=g^{\prime} \cdot f_{\ell}$ for some $g^{\prime} \in G$. If

$$
g^{\prime}=\left|\begin{array}{cc}
\frac{\alpha^{\prime}}{-\beta^{\prime}} & \frac{\beta^{\prime}}{\alpha^{\prime}}
\end{array}\right| \text { then } g^{\prime} f_{\ell} g^{-1}=\frac{i \ell}{2}\left|\begin{array}{cc}
2\left|\alpha^{\prime}\right|^{2}-1 & -2 \alpha^{\prime} \beta^{\prime} \\
-2 \overline{\alpha^{\prime}} \overline{\beta^{\prime}} & 1-2\left|\alpha^{\prime}\right|^{2}
\end{array}\right| \text {. }
$$

Now since $e\left(g, g^{\prime} f_{\ell} g^{-1}\right)=e\left(g^{\prime-1} g g^{\prime}, f_{\ell}\right)$ we apply Lemma 7.2 to obtain $e\left(g, g^{\prime} f_{\ell} g^{-1}\right)=\left(\alpha^{\prime \prime}\right)^{2 \ell}$ where

$$
g^{\prime-1} g g^{\prime}=\left|\begin{array}{rr}
\alpha^{\prime \prime} & \beta^{\prime \prime} \\
-\overline{\beta^{\prime \prime}} & \overline{\alpha^{\prime \prime}}
\end{array}\right| .
$$

A short calculation shows that

$$
\alpha^{\prime \prime}=\alpha\left|\alpha^{\prime}\right|^{2}+\bar{\alpha}\left|\beta^{\prime}\right|^{2}+\bar{\beta}\left(\alpha^{\prime} \beta^{\prime}\right)-\beta\left(\overline{\alpha^{\prime} \beta^{\prime}}\right) .
$$

But $\left|\beta^{\prime}\right|^{2}=1-\left|\alpha^{\prime}\right|^{2}$ so

$$
\begin{aligned}
\alpha^{\prime \prime} & =\left(\frac{\alpha-\bar{\alpha}}{2}\right)\left(2\left|\alpha^{\prime}\right|^{2}-1\right)+\bar{\beta}\left(\alpha^{\prime} \beta^{\prime}\right)-\beta\left(\overline{\alpha^{\prime} \beta^{\prime}}\right)+\left(\frac{\alpha+\bar{\alpha}}{2}\right) \\
& =\frac{i}{2 \ell}\left(\left|\begin{array}{cc}
\frac{\bar{\alpha}-\alpha}{2} & -\beta \\
\bar{\beta} & \frac{\alpha-\bar{\alpha}}{2}
\end{array}\right|, \quad \frac{i \ell}{2}\left|\begin{array}{cc}
2\left|\alpha^{\prime}\right|^{2}-1 & -2 \alpha^{\prime} \beta^{\prime} \\
-2 \overline{\alpha^{\prime} \beta^{\prime}} & 1-2\left|\alpha^{\prime}\right|^{2}
\end{array}\right|\right)+\frac{\alpha+\bar{\alpha}}{2} \\
& =\frac{\operatorname{tr} g}{2}+\frac{i}{2 \ell}(C(g), X) .
\end{aligned}
$$


Thus

$$
\begin{aligned}
e(g, X) & =\left(\alpha^{\prime \prime}\right)^{2 \ell} \\
& =\left(\frac{\operatorname{tr} g}{2}+\frac{i}{2 \ell}(C(g), X)\right)^{2 \ell}
\end{aligned}
$$

LEMMA 7.6. $C: G \rightarrow \mathfrak{g}$ has the following properties

(a) $C(e)=0$;

(b) $C$ is a $G$-map under the actions of $G$ on $G$ and $\mathfrak{g}$ by conjugation and the adjoint action respectively;

(c) $\operatorname{Im} C=\left\{\left.X \in \mathfrak{g}|| X\right|^{2}=(X, X) \leq 4\right\}$.

PROOF. (a) and (b) are easily checked. Now for

$$
g=\left|\begin{array}{cc}
e^{i \theta} & 0 \\
0 & e^{-i \theta}
\end{array}\right|
$$

we have

$$
C(g)=\left|\begin{array}{cc}
-i \sin \theta & 0 \\
0 & i \sin \theta
\end{array}\right|
$$

so that

$$
|C(g)|^{2}=4 \sin ^{2} \theta \leq 4
$$

Let $\mathscr{M}_{\ell} \in \Omega_{\ell}$ be the extremal orbit of $\rho_{\ell}$.

PROPOSITION 7.7. Let $m_{1}, m_{2} \in \mathscr{M}_{\ell}$ and $\Phi\left(m_{i}\right)=f_{i}, i=1,2$ with $f_{i} \in \mathscr{O}_{\ell}$. Then

$$
\left|\left\langle m_{1}, m_{2}\right\rangle\right|^{2}=\left(\frac{1}{2}+\frac{1}{2 \ell^{2}}\left(f_{1}, f_{2}\right)\right)^{2 \ell} .
$$

PROOF. Let

$$
g=\left|\begin{array}{cc}
\alpha & \beta \\
-\bar{\beta} & \bar{\alpha}
\end{array}\right| \in G
$$

A short computation shows that

$$
\left(g f_{\ell} g^{-1}, f_{\ell}\right)=\ell^{2}\left(2|\alpha|^{2}-1\right) .
$$

Thus

$$
|\alpha|^{2}=\frac{1}{2}+\frac{1}{2 \ell^{2}}\left(g f_{\ell} g^{-1}, f_{\ell}\right)
$$


Let $m_{\ell} \in \mathscr{M}_{\ell}$ such that $\Phi\left(m_{\ell}\right)=f_{\ell}$ (for example, we could take $m_{\ell}=\psi_{-\ell}$ by Lemma 7.1). Then $\left\langle g \cdot m_{\ell}, m_{\ell}\right\rangle=e\left(g, f_{\ell}\right)=\alpha^{2 \ell}$. Thus

$$
\begin{aligned}
\left|\left\langle g \cdot m_{\ell}, m_{\ell}\right\rangle\right|^{2} & =\left(|\alpha|^{2}\right)^{2 \ell} \\
& =\left(\frac{1}{2}+\frac{1}{2 \ell^{2}}\left(g f_{\ell} g^{-1}, f_{\ell}\right)\right)^{2 \ell} .
\end{aligned}
$$

For any other $g^{\prime} \in G$, we thus have

$$
\left|\left\langle g \cdot m_{\ell}, g^{\prime} \cdot m_{\ell}\right)\right|^{2}=\left(\frac{1}{2}+\frac{1}{2 \ell^{2}}\left(g f_{\ell} g^{-1}, g^{\prime} f_{\ell} g^{\prime-1}\right)\right)^{2 \ell} .
$$

But then $g f_{\ell} g^{-1}=f_{1}$ and $g^{\prime} f_{\ell} g^{\prime-1}=f_{2}$ are arbitrary elements of $\mathscr{O}_{\ell}$ and $m_{1}=g \cdot m_{\ell}, m_{2}=g^{\prime} \cdot m_{\ell}$ with $\Phi\left(m_{i}\right)=f_{i}$.

COROLLARY 7.8. If $m_{1}, m_{2} \in \mathscr{M}_{\ell}$ and $\Phi\left(m_{i}\right)=f_{i} \in \mathscr{O}_{\ell}, i=1,2$, then

(a) $\left|\left\langle m_{1}, m_{2}\right\rangle\right|^{2}=1$ if and only if $f_{1}=f_{2}$;

(b) $\left|\left\langle m_{1}, m_{2}\right\rangle\right|^{2}=0$ if and only if $f_{1}=-f_{2}$;

(c) $\left|\left\langle m_{1}, m_{2}\right\rangle\right|^{2}=(1 / 2)^{2 \ell}$ if and only if $\left(f_{1}, f_{2}\right)=0$.

PROOF. These statements follow immediately from the previous proposition and the fact that for $f_{1}, f_{2} \in \mathscr{O}_{\ell}$

$$
-\ell^{2} \leq\left(f_{1}, f_{2}\right) \leq \ell^{2}
$$

since $\mathscr{O}_{\ell}$ is a sphere of radius $\ell$.

COROLlaRY 7.9. For any $m \in \mathscr{M}_{\ell}$, if $m^{\perp}=\{v \in V \mid\langle v, m\rangle=0\}$ then $m^{\perp} \cap \mathscr{M}_{\ell}$ is a circle $S^{1}$.

PROOF. Corollary 7.8 shows that if $\left\langle m, m^{\prime}\right\rangle=0$ with $m^{\prime} \in \mathscr{M}_{\ell}$, then $\Phi\left(m^{\prime}\right)=$ $-\Phi(m)$. But then $m^{\prime}$ is determined uniquely up to a scalar of modulus one.

We now turn to the space of functions $A_{\rho}=A_{\ell}$ on $\mathscr{O}_{\ell}$ and the $*$-product on this space. For $g \in G$, let $e_{g}(f)=e(g, f)$. Then the functions $e_{g}, g \in G$ span $A_{\ell}$ and

$$
e_{g_{1}} * e_{g_{2}}=e_{g_{1} g_{2}} \text {. }
$$

Any $X \in \mathfrak{g}$ may be regarded as a function on $\mathfrak{g}^{*}$ and so also on $\mathscr{O}_{\ell}$. If $\phi$ is any polynomial then $\phi(X)$ is the corresponding element in $S(\mathfrak{g})$, which we also 
view as a function on $\mathfrak{g}^{*}$ and so on $\mathscr{O}_{\ell}$. Note that if $g \in G$ and $C(g)=Y \in \mathfrak{g}$, then Theorem 7.5 shows that

$$
e_{g}=\left(\frac{\operatorname{tr} g}{2}+\frac{i}{2 \ell} Y\right)^{2 \ell}
$$

which is a polynomial function on $\mathscr{O}_{\ell}$.

PROPOSITION 7.10. If $X \in \mathfrak{g}$ and $\phi$ is a polynomial, with $\phi(X) \in A_{\ell}$, then

$$
X * \phi(X)=X \phi(X)-\frac{\left(X^{2}-(2 \ell r)^{2}\right)}{2 \ell} \frac{d}{d X} \phi(X)
$$

where $r=|X| / 2$.

PROOF. We will prove this for

$$
X=\left|\begin{array}{cc}
i x & 0 \\
0 & -i x
\end{array}\right| \in \mathfrak{g} .
$$

The general case will follow since an arbitrary element of $\mathfrak{g}$ is conjugate (under $G)$ to such an $X$. Let

$$
g_{t}=\exp t X=\left|\begin{array}{cc}
e^{i t x} & 0 \\
0 & e^{-i t x}
\end{array}\right| \in G .
$$

Then

$$
e_{g_{1}} * e_{g_{s}}=e_{g_{1+s}}
$$

where $t, s \in \mathbb{R}$ and

$$
e_{g_{t}}=\left(\cos t x+\frac{i}{2 \ell} C\left(g_{t}\right)\right)^{2 \ell}
$$

with

$$
C\left(g_{t}\right)=\left|\begin{array}{cc}
-i \sin t x & 0 \\
0 & i \sin t x
\end{array}\right|=-\frac{\sin t x}{x} X .
$$

We differentiate both sides of (7.1) with respect to $t$ and set $t=0$

$$
\begin{aligned}
2 \ell\left(-\frac{i}{2 \ell} X\right) & *\left(\cos s x-\frac{i \sin s x}{2 \ell x} X\right)^{2 \ell} \\
& =2 \ell\left(\cos s x-\frac{i \sin s x}{2 \ell x} X\right)^{2 \ell-1}\left(-x \sin s x-\frac{i \cos s x}{2 \ell} X\right) .
\end{aligned}
$$


Then

$$
\begin{aligned}
& X *\left(\cos s x-\frac{i \sin s x}{2 \ell x} X\right)^{2 \ell} \\
& =\left(\cos s x-\frac{i \sin s x}{2 \ell x} X\right)^{2 \ell-1}(-2 \ell i x \sin s x+\cos s x X) \\
& =\left(\cos s x-\frac{i \sin s x}{2 \ell x} X\right)^{2 \ell-1} \\
& \quad \times\left[X\left(\cos s x-\frac{i \sin s x}{2 \ell x} X\right)-\left(X^{2}-(2 \ell x)^{2}\right)\left(-\frac{i \sin s x}{2 \ell x}\right)\right] \\
& =X\left(\cos s x-\frac{i \sin s x}{2 \ell x}\right)^{2 \ell}-\frac{\left(X^{2}-(2 \ell x)^{2}\right)}{2 \ell} \frac{d}{d X}\left(\cos s x-\frac{i \sin s x}{2 \ell x} X\right)^{2 \ell}
\end{aligned}
$$

Thus

$$
X * e_{g_{s}}=X e_{g_{s}}-\frac{\left(X^{2}-(2 \ell x)^{2}\right)}{2 \ell} \frac{d}{d X} e_{g_{s}} .
$$

Now if $\phi$ is any polynomial such that $\phi(X) \in A_{\ell}$, then $\phi(X)$ is in the span of the $e_{g_{s}}$ as $s \in \mathbb{R}$, so that

$$
X * \phi(X)=X \phi(X)-\frac{\left(X^{2}-(2 \ell x)^{2}\right)}{2 \ell} \frac{d}{d X} \phi(X) .
$$

Note that $(X, X)=4 x^{2}$ so $x=|X| / 2=r$.

The above proposition shows that the $*$-product we have constructed agrees in the case of $G=S U(2)$ with that obtained by Moreno and Ortega-Navarro [14] on $S^{2}$.

COROLlaRY 7.11. $X_{1} * X_{1}+X_{2} * X_{2}+X_{3} * X_{3}=\ell(\ell+1)$.

PROOF. From the previous proposition, we have

$$
X_{i} * X_{i}=X_{i}^{2}-\frac{\left(X_{i}^{2}-\ell^{2}\right)}{2 \ell}=X_{i}^{2}\left(\frac{2 \ell-1}{2 \ell}\right)+\frac{\ell}{2}
$$

Thus

$$
\begin{aligned}
\sum_{i=1}^{3} X_{i} * X_{i} & =\left(\frac{2 \ell-1}{2 \ell}\right) \sum_{i=1}^{3} X_{i}^{2}+\frac{3 \ell}{2} \\
& =\left(\frac{2 \ell-1}{2 \ell}\right) \ell^{2}+\frac{3 \ell}{2} \\
& =\ell(\ell+1) .
\end{aligned}
$$


The inner product $\langle,\rangle_{1}$ on $A_{\ell}$ is determined by the kernel $N^{\prime}\left(f, f^{\prime}\right)$ on $\mathscr{O}_{\ell}$ (see Section 5) and from Proposition 7.7 we see immediately that

$$
N^{\prime}\left(f, f^{\prime}\right)=\left(\frac{1}{2}+\frac{1}{2 \ell^{2}}\left(f, f^{\prime}\right)\right)^{2 \ell}
$$

for $f, f^{\prime} \in \mathscr{O}_{\ell}$. The $*$-product is determined by the triple kernel $B_{1}^{\prime}\left(f_{1}, f_{2}, f_{3}\right)$ with respect to $\langle,\rangle_{1}$ which we now determine.

THEOREM 7.12. For $f_{1}, f_{2}, f_{3} \in \mathscr{O}_{\ell}$, let $\Delta=\Delta\left(f_{1}, f_{2}, f_{3}\right)$ be a geodesic triangle through $f_{1}, f_{2}, f_{3}$. Then

$$
B_{1}^{\prime}\left(f_{1}, f_{2}, f_{3}\right)=\left|\cos \frac{\theta_{1}}{2} \cos \frac{\theta_{2}}{2} \cos \frac{\theta_{3}}{2}\right|^{2 \ell} e^{-\left|\Delta\left(f_{1}, f_{2}, f_{3}\right)\right|}
$$

where $\theta_{1}, \theta_{2}, \theta_{3}$ are the angles subtended at the origin 0 by the arcs $f_{2} f_{3}, f_{3} f_{1}$ and $f_{1} f_{2}$ respectively, and $\left|\Delta\left(f_{1}, f_{2}, f_{3}\right)\right|$ is the (signed) area of the triangle $\Delta$ with respect to the 2 -form $\omega$.

PROOF. Let $m_{i} \in \mathscr{M}_{\ell} \subset \Omega_{\ell}$ such that $\Phi\left(m_{i}\right)=f_{i}, i=1,2,3$. Then

$$
\begin{aligned}
B_{1}^{\prime}\left(f_{1}, f_{2}, f_{3}\right) & =B_{1}\left(m_{1}, m_{2}, m_{3}\right) \\
& =\left\langle m_{1}, m_{2}\right\rangle\left\langle m_{2}, m_{3}\right\rangle\left\langle m_{3}, m_{1}\right)
\end{aligned}
$$

from Theorem 3.11 .

Thus using Proposition 3.5

$$
\begin{aligned}
\left|B_{1}^{\prime}\left(f_{1}, f_{2}, f_{3}\right)\right|^{2} & =\left|\left\langle m_{1}, m_{2}\right\rangle\right|^{2}\left|\left\langle m_{2}, m_{3}\right\rangle\right|^{2}\left|\left\langle m_{3}, m_{1}\right\rangle\right|^{2} \\
& =N\left(m_{1}, m_{2}\right) N\left(m_{2}, m_{3}\right) N\left(m_{3}, m_{1}\right) \\
& =N^{\prime}\left(f_{1}, f_{2}\right) N^{\prime}\left(f_{2}, f_{3}\right) N^{\prime}\left(f_{3}, f_{1}\right) \\
& =\left(\frac{1}{2}+\frac{\cos \theta_{1}}{2}\right)^{2 \ell}\left(\frac{1}{2}+\frac{\cos \theta_{2}}{2}\right)^{2 \ell}\left(\frac{1}{2}+\frac{\cos \theta_{3}}{2}\right)^{2 \ell} .
\end{aligned}
$$

Thus

$$
\left|B_{1}^{\prime}\left(f_{1}, f_{2}, f_{3}\right)\right|=\left|\cos \frac{\theta_{1}}{2} \cos \frac{\theta_{2}}{2} \cos \frac{\theta_{3}}{2}\right|^{2 \ell}
$$

and we need only show that

$$
\arg B_{1}^{\prime}\left(f_{1}, f_{2}, f_{3}\right)=-\left|\Delta\left(f_{1}, f_{2}, f_{3}\right)\right|
$$


Let the $\operatorname{arcs} f_{2} f_{3}, f_{3} f_{1}$ and $f_{1} f_{2}$ on $\mathscr{O}_{\ell}$ be denoted by $\gamma_{1}, \gamma_{2}$ and $\gamma_{3}$ where

$$
\begin{array}{ll}
\gamma_{1}(t)=\exp t Y_{1} \cdot f_{2} & 0 \leq t \leq 1 \\
\gamma_{2}(t)=\exp t Y_{2} \cdot f_{3} & 0 \leq t \leq 1 \\
\gamma_{3}(t)=\exp t Y_{3} \cdot f_{1} & 0 \leq t \leq 1
\end{array}
$$

and $Y_{1}, Y_{2}, Y_{3} \in \mathfrak{g}$. The condition that the $\gamma_{i}$ be geodesics is equivalent to the conditions

$$
0=f_{2}\left(Y_{1}\right)=f_{3}\left(Y_{2}\right)=f_{1}\left(Y_{3}\right) .
$$

We now choose $m_{1}, \ldots, m_{3}$ as above more carefully. Begin with an arbitrary but fixed $m_{1} \in \Phi^{-1}\left(f_{1}\right)$, and define the curve $\gamma_{3}^{\prime}$ in $\mathscr{M}_{\ell}$ by

$$
\gamma_{3}^{\prime}(t)=\exp t Y_{3} \cdot m_{1}, \quad 0 \leq t \leq 1
$$

with final endpoint $m_{2}=\exp Y_{3} \cdot m_{1}$. Then define

$$
\gamma_{1}^{\prime}(t)=\exp t Y_{1} \cdot m_{2}, \quad 0 \leq t \leq 1
$$

with final endpoint $m_{3}=\exp Y_{1} \cdot m_{2}$. Then define

$$
\gamma_{2}^{\prime}(t)=\exp t Y_{2} \cdot m_{3}, \quad 0 \leq t \leq 1
$$

and call its endpoint $m_{4}=\exp Y_{2} \cdot m_{3}$. Then by Lemma 4.2,

$$
\Phi \circ \gamma_{i}^{\prime}=\gamma_{i} \quad i=1, \ldots, 3
$$

and $\Phi\left(m_{i}\right)=f_{i}, i=1, \ldots, 3$. Since $\Phi\left(m_{4}\right)=f_{1}$, Proposition 4.9 implies that

$$
m_{1}=e^{i \tau} m_{4}
$$

for some $\tau \in \mathbb{R}$. Thus define

$$
\gamma_{4}^{\prime}(t)=e^{i t} m_{4} \quad 0 \leq t \leq \tau .
$$

Denote the closed curve $\gamma_{1}+\gamma_{2}+\gamma_{3}$ in $\mathscr{O}_{\ell}$ by $\gamma$ and the closed curve $\gamma_{1}^{\prime}+\gamma_{2}^{\prime}+\gamma_{4}^{\prime}+\gamma_{3}^{\prime}$ by $\gamma^{\prime}$. Then $\gamma^{\prime}$ lies over $\gamma$ in the $S^{1}$ bundle $\Phi: \mathscr{M}_{\ell} \rightarrow \mathscr{O}_{\ell}$. Suppose that $\gamma$ is oriented positively with respect to the 2 -form $\omega$ on $\mathscr{O}_{\ell}$. Recall from Lemma 5.1 and the remarks following it that $\omega$ is the curvature form for the connection 1-form $\theta$ on $\mathscr{M}_{\ell}$. Thus

$$
\begin{aligned}
\left|\Delta\left(f_{1}, f_{2}, f_{3}\right)\right| & =\int_{\Delta} \omega \\
& =\int_{\gamma^{\prime}} \theta .
\end{aligned}
$$


We claim that

$$
0=\int_{\gamma_{1}^{\prime}} \theta=\int_{\gamma_{2}^{\prime}} \theta=\int_{\gamma_{3}^{\prime}} \theta .
$$

For let $m=\exp s X_{1} \cdot m_{2}, 0 \leq s \leq 1$, be on $\gamma_{1}^{\prime}$ say. Then a tangent vector to $\gamma_{1}^{\prime}$ at $m$ is $\eta=X_{1} \cdot m$ and so

$$
\begin{aligned}
\theta_{m}(\eta) & =\operatorname{Im}\langle\eta, m\rangle \\
& =\operatorname{Im}\left\langle X_{1} \cdot m, m\right\rangle \\
& =\Phi(m)\left(X_{1}\right) \\
& =\Phi\left(m_{2}\right)\left(\exp -s X_{1} \cdot X_{1}\right) \\
& =f_{2}\left(X_{1}\right) \\
& =0 .
\end{aligned}
$$

This proves the claim and shows that

$$
\begin{aligned}
\left|\Delta\left(f_{1}, f_{2}, f_{3}\right)\right| & =\int_{\gamma_{4}^{\prime}} \theta \\
& =\int_{0}^{\tau} \operatorname{Im}\left\langle i e^{i t} m_{4}, e^{i t} m_{4}\right\rangle d t \\
& =i \tau
\end{aligned}
$$

On the other hand

$$
\begin{aligned}
\arg B_{1}^{\prime}\left(f_{1}, f_{2}, f_{3}\right)=\arg \left\langle m_{1}, m_{2}\right\rangle & +\arg \left\langle m_{2}, m_{3}\right\rangle \\
& +\arg \left\langle m_{3}, m_{4}\right\rangle+\arg \left\langle m_{4}, m_{1}\right\rangle .
\end{aligned}
$$

We now claim that

$$
\arg \left\langle m_{1}, m_{2}\right\rangle=\arg \left\langle m_{2}, m_{3}\right\rangle=\arg \left\langle m_{3}, m_{4}\right\rangle=0
$$

To see this, it suffices to consider the model of $V_{\ell}$ described at the beginning of this section. Suppose $m_{1}=\psi_{-\ell}$. Then from Lemma 7.1,

$$
\Phi_{\ell}\left(\psi_{-\ell}\right)=\ell X_{3}^{*}=f_{1} .
$$

Then since $f_{1}\left(Y_{3}\right)=0$, we may take $Y_{3}$ to be

$$
Y_{3}=s\left|\begin{array}{cc}
0 & 1 \\
-1 & 0
\end{array}\right|, \quad s \in \mathbb{R} \text {. }
$$


by conjugating by an element of $G$ if necessary. But then $\exp t Y_{3}$ has the form

$$
\left|\begin{array}{cc}
\cos s t & \sin s t \\
-\sin s t & \cos s t
\end{array}\right|
$$

and thus we find that

$$
\left\langle\exp t Y_{3} \cdot m_{1}, m_{1}\right\rangle \in R
$$

for all $t \in \mathbb{R}$ so that $\left\langle m_{1}, m_{2}\right\rangle \in R$. This proves the second claim since the other two cases are conjugate to this one. We thus have

$$
\begin{aligned}
\arg B_{1}^{\prime}\left(f_{1}, f_{2}, f_{3}\right) & =\arg \left\langle m_{4}, m_{1}\right\rangle \\
& =-i \tau \\
& =-\left|\Delta\left(f_{1}, f_{2}, f_{3}\right)\right|
\end{aligned}
$$

as required.

If $\gamma$ is not oriented positively then we apply the argument to the triangle $\Delta\left(f_{1}, f_{3}, f_{2}\right)$ to show that

$$
\begin{aligned}
\arg B_{1}^{\prime}\left(f_{1}, f_{3}, f_{2}\right) & =-\left|\Delta\left(f_{1}, f_{3}, f_{2}\right)\right| \\
& =\left|\Delta\left(f_{1}, f_{2}, f_{3}\right)\right| .
\end{aligned}
$$

But $B_{1}^{\prime}\left(f_{1}, f_{3}, f_{2}\right)=\overline{B_{1}^{\prime}\left(f_{1}, f_{2}, f_{3}\right)}$ so we are done.

Note that the geodesic triangle $\Delta$ was not uniquely specified in the above theorem. This is due to the integrality of the 2 -form $\omega$ on $\mathscr{O}_{\ell}$, that is

$$
\int_{\mathscr{O}_{\ell}} \omega
$$

is a multiple of $2 \pi$. In fact we may view this as a consequence of Theorem 7.12 if we wish. Of course it is the existence of the $S^{1}$ bundle with connection over $\mathscr{O}_{\ell}$ that is the real reason for the integrality of $\mathscr{O}_{\ell}$ (see for example Kostant [11]).

It turns out that the function $B_{1}^{\prime}\left(f_{1}, f_{2}, f_{3}\right)$ has a simple radial dependence. Since $f_{i} \in \mathscr{O}_{\ell}$, let

$$
x_{i}=\frac{f_{i}}{\ell} \in \mathscr{O}_{1}, \quad i=1,2,3 .
$$

If $\Delta\left(f_{1}, f_{2}, f_{3}\right)$ is a geodesic triangle through $f_{1}, f_{2}$ and $f_{3}$, let $\Delta^{\prime}\left(x_{1}, x_{2}, x_{3}\right)$ be the corresponding triangle on $\mathscr{O}_{1}$. Then it is an immediate consequence of (5.1) that

$$
\left|\Delta\left(f_{1}, f_{2}, f_{3}\right)\right|=\ell\left|\Delta\left(x_{1}, x_{2}, x_{3}\right)\right| .
$$


We may thus rewrite the formula of Theorem 7.12 as

$$
B_{1}^{\prime}\left(\ell x_{1}, \ell x_{2}, \ell x_{3}\right)=\left(\left|\cos \frac{\theta_{1}}{2} \cos \frac{\theta_{2}}{2} \cos \frac{\theta_{3}}{2}\right| e^{-i\left|\Delta\left(x_{1}, x_{2}, x_{3}\right)\right| / 2}\right)^{2 \ell} .
$$

Thus it suffices to study $B_{1}^{\prime}$ on the unit sphere $\mathscr{O}_{1}$.

THEOREM 7.13. For $x_{1}, x_{2}, x_{3} \in \mathscr{O}_{1}$,

$$
\left.4 B_{1}^{\prime}\left(x_{1}, x_{2}, x_{3}\right)=1+\left(x_{1}, x_{2}\right)+x_{2}, x_{3}\right)+\left(x_{3}, x_{1}\right)-i\left(x_{1}, x_{2} \times x_{3}\right)
$$

where $x_{2} \times x_{3}$ is the cross product of $x_{2}$ and $x_{3}$, and is identified with $\left[x_{2}, x_{3}\right]$ under $\mathbf{g}^{*} \simeq \mathbf{g}$.

PROOF. This is a result of certain formulae in spherical trigonometry. If $\Delta$ is the spherical triangle whose sides are all no greater than $\pi$ with vertices $x_{1}, x_{2}$ and $x_{3}$ and opposite sides $\mathrm{a}, \mathrm{b}$ and $\mathrm{c}$ respectively then we find the following formula from Todhunter [17, Section 138]

$$
\tan \frac{1}{2} E=\frac{2 n}{1+\cos a+\cos b+\cos c}
$$

where $E$ is the spherical excess of $\Delta$, and is equal to the absolute value of the area in our case, and $2 n$ is the sine of the trihedral angle subtended by the triangle at the origin. From remarks made in [17, Section 51] of the same reference we may conclude that in our notation

$$
2 n=\left(x_{1}, x_{2} \times x_{3}\right)
$$

that is, it is the triple-product of $x_{1}, x_{2}$ and $x_{3}$. We also find there that

$$
4 n^{2}=1-\cos ^{2} a-\cos ^{2} b-\cos ^{2} c+2 \cos a \cos b \cos c .
$$

From this we may easily calculate that

$$
(2 n)^{2}+(1+\cos a+\cos b+\cos c)^{2}=\left(4 \cos \frac{a}{2} \cos \frac{b}{2} \cos \frac{c}{2}\right)^{2} .
$$

It follows that

$$
1+\cos a+\cos b+\cos c-i 2 n=4 \cos \frac{a}{2} \cos \frac{b}{2} \cos \frac{c}{2} e^{-i E / 2}
$$

Our result follows. 
We cannot resist extracting a few more relevant formulae from Todhunter. From Section 154 we get Keogh's theorem, stating that

$$
\sin \frac{1}{2} E=2 n^{\prime}
$$

where $2 n^{\prime}$ is the sine of the trihedral angle subtended by the triangle whose vertices are in the midpoints of $\Delta$. Combining this with (7.2), we have

$$
4 \cos \frac{1}{2} a \cos \frac{1}{2} b \cos \frac{1}{2} c=\frac{2 n}{2 n^{\prime}} .
$$

This is a purely geometric interpretation of $\left|B_{1}^{\prime}\right|$. Another one is

$$
4 \cos \frac{1}{2} a \cos \frac{1}{2} b \cos \frac{1}{2} c=\cos s+\cos (s-a)+\cos (s-b)+\cos (s-c)
$$

where $2 s=a+b+c$, found in [17, Section 119].

It seems an interesting program to extend these results to more general compact groups.

\section{Acknowledgements}

This work was supported by the National Science and Engineering Research Council of Canada.

\section{References}

[1] D. Amal, M. Cahen and S. Gutt, 'Representatins of compact Lie groups and quantization by deformation', Note à paraître à l'Académie des Sciences de Belgique (1988).

[2] D. Arnal and J. C. Cortet, 'Nilpotent Fourier transform and applications', Lett. Math. Phys. 9 (1985), 25-34.

[3] D. Arnal and J. Ludwig, 'La convexité de l'application moment d'un groupe de Lie', $J$. Funct. Anal. 105 (1992), 256-300.

[4] H. Figueroa, J. M. Gracia-Bondía and J. C. Várilly, 'Moyal quantization with compact symmetry groups and noncommutative harmonic analysis', J. Math. Phys. 31 (1990), 2664-2671.

[5] S. Gutt, 'An explicit *-product on the cotangent bundle of a Lie group', Lett. Math. Phys. 7 (1983), 249-258.

[6] S. Helgason, Groups and geometric analysis (Academic Press, Orlando, 1984). 
[7] R. Howe, G. Ratcliff and N. Wildberger, 'Symbol mappings for certain nilpotent groups', in: Lie Group Representations III, Proceedings U. of Maryland, volume 1077 of Lecture Notes in Mathematics (Springer-Verlag, Berlin, 1984) pp. 288-320.

[8] M. S. Khalgui, 'Charactères des groupes de Lie', J. Funct. Anal 47 (1982), 64-77.

[9] A. A. Kirillov, 'Unitary representations of nilpotent Lie groups', Russian. Math. Surveys 17 (1962), 53-103.

[10] A. Knapp and B. Speh, 'Status of classification of irreducible unitary representations', in: Harmonic Analysis Proc., Minneapolis, volume 908 of Lecture Notes in Mathematics (Springer, Berlin, 1982).

[11] B. Kostant, 'Quantization and unitary representations', in: Lectures in modern analysis III (ed. C. T. Taam), volume 170 of Lecture Notes in Mathematics (Springer, Berlin, 1970) pp. 87-208.

[12] - 'On convexity, the Weyl group and the Iwasawa decomposition', Ann. Sci. École Norm. Sup. 6 (1973), 413-455.

[13] A. Lichnerowicz, 'Existence and invariance of twisted products on a symplectic manifold', Lett. Math. Phys. 5 (1981), 117-126.

[14] C. Moreno and P. Ortega-Navarro, '*-products on $D^{\prime}(\mathbb{C}), S^{2}$ and related spectral analysis', Lett. Math. Phys. 7 (1983), 181-193.

[15] T. Sherman, 'Fourier analysis on the sphere', Trans. Amer. Math. Soc. 209 (1975), 1-31.

[16] J. M. Souriau, Structure des systèmes dynamiques (Dunod, Paris, 1970).

[17] I. Todhunter, Spherical Trigonometry for the use of colleges and schools (Macmillan, London, 1914).

[18] V. Varadarajan, Lie algebras and their representations (Prentice-Hall, Englewood Cliffs, 1974).

[19] J. C. Várilly and J. M. Gracia-Bondía, 'The Moyal representation for spin', Ann. Physics 190 (1989), 107-148.

[20] N. Vilenkin, Special functions and the theory of group representations (Amer. Math. Society, Providence, 1968).

[21] D. Vogan, 'The unitary dual of $G L(n)$ over an archimedean field', Invent. Math. 83 (1986), 449-505.

[22] N. Wildberger, Quantization and harmonic analysis on nilpotent groups (Ph.D. Thesis, Yale University, 1983).

[23] - 'Convexity and unitary representations of nilpotent Lie groups', Invent. Math. 98 (1989), 281-292.

[24] - 'The moment map of a Lie group representation', Trans. Amer. Math. Soc. 330 (1992), 257-268.

Department of Pure Mathematics

University of N. S. W.

P. O. Box 1

Kensington N. S. W. 2033

Australia 